Supplement of Atmos. Chem. Phys., 21, 13655-13666, 2021

https://doi.org/10.5194/acp-21-13655-2021-supplement

(C) Author(s) 2021. CC BY 4.0 License.

(c) (i)

Atmospheric

Chemistry

and Physics

Supplement of

\title{
A mass-balance-based emission inventory of non-methane volatile organic compounds (NMVOCs) for solvent use in China
}

Ziwei Mo et al.

Correspondence to: Bin Yuan (byuan@jnu.edu.cn) and Min Shao (mshao@pku.edu.cn)

The copyright of individual parts of the supplement might differ from the article licence. 


\section{Contents}

Text S1. Source profiles used in this study.

Table S1-S5 Data source for VOC content of coating, ink, adhesive, insecticide, cleaner and personal care.

Table S6. Five-tier evaluation system for uncertainty in activity data (Wei et al., 2011a).

Table S7. The parameters for calculating emission of various solvent product.

Table S8. Proxy variables for allocating national emissions to provincial level.

Table S9. List of VOCs and S/IVOCs and their MIR and SOA yield values used in this study.

Table S10. Market share of NMVOCs control technologies and their control efficiency.

Table S11. Annual value of production for organic exhaust gas treatment industry, percentage with NMVOCs treatment facilities and effective control efficiency during 2000-2017.

Table S12. Solvent use emissions from various categories in 2000-2017 (Tg).

Table S13. Source categories, emission factors and activity data source for solvent use NMVOC emissions in different studies.

Figure S1. (a) The linear relationship between formaldehyde-type adhesive consumption and the artificial board yield for estimating consumption of formaldehyde-type adhesive, and (b) consumption of formaldehyde-type adhesive during 2000-2017.

Figure S2. Pesticide production, import and export data during 2000-2017 in China.

Figure S3-S12. The procedure of source profiles for coating, ink, adhesive and herbicide.

Figure S13. NMVOCs group emission from various solvent categories in 2017.

Figure S14. Comparisons of (a) NMVOCs emissions and (b) their increasing percentage compared to 2000 from solvent use (this study) and other sources (MEIC). 


\section{Text S1. Source profiles used in this study.}

Source profiles of solvents use used in this study are obtained by combining the domestic and foreign profiles. This compilation of the profiles can make use of the local measurements in China as well as including more comprehensive species, such as S/IVOCs being listed in the foreign profiles. The procedure involves four steps as shown in the following flow chart.

\section{Step 1: Averaging the profiles}

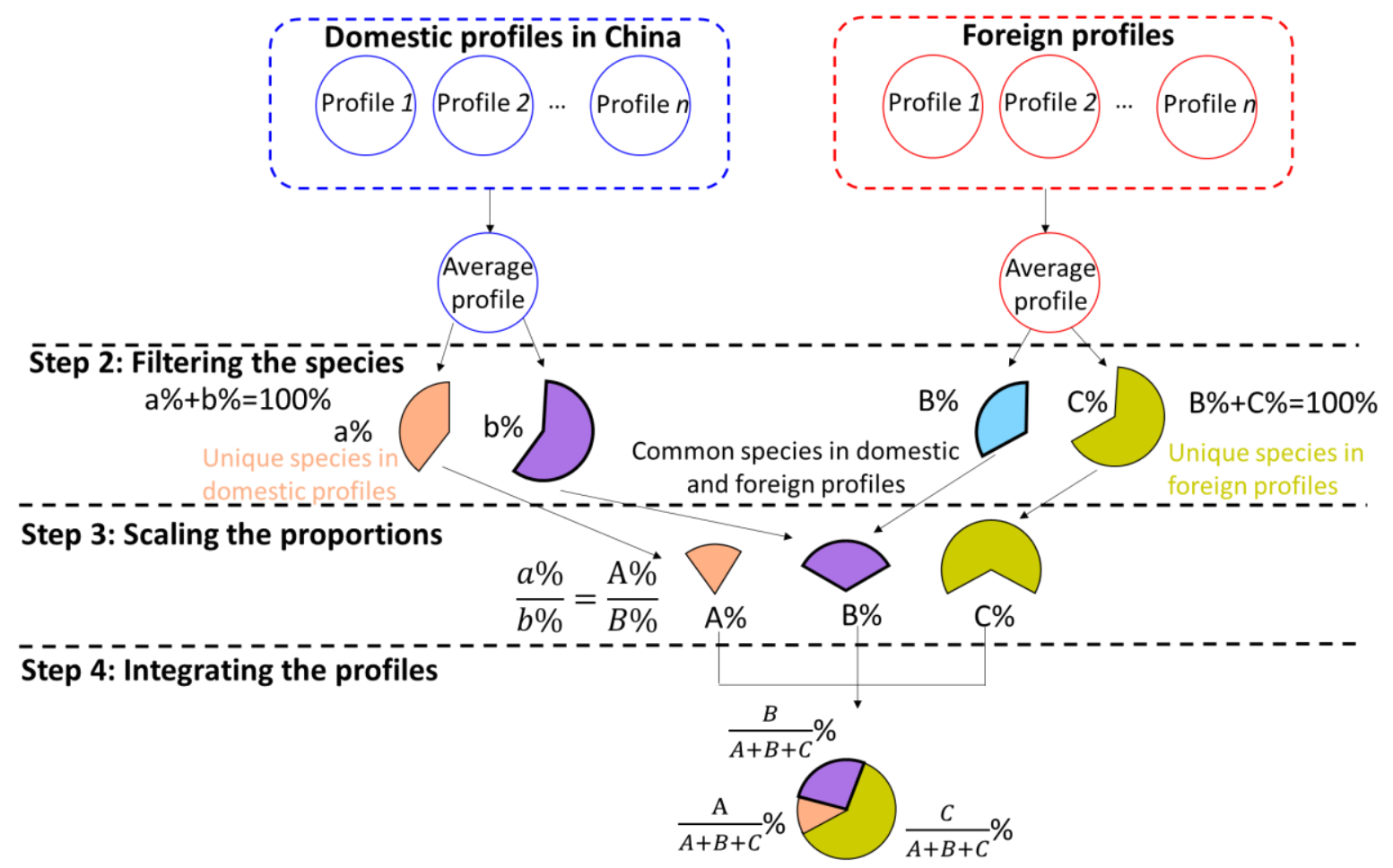

Step 1: A new domestic or foreign profile is formed by averaging weight percentages of NMVOCs groups from multiple source profiles. If some source profiles have OVOC, the treatment of OVOC followed the methods in Wu and Xie (2017) and Li et al. (2014) by averaging the NMHCs and OVOCs proportions, respectively.

Step 2: Common species in the domestic and foreign profiles are identified. Common species may account for different proportions in the domestic and foreign profiles. For example, common species account for $\mathrm{b} \%$ in the average domestic profile, while $\mathrm{B} \%$ in the average foreign profile. The remaining unique species account for $\mathrm{a} \%$ in the domestic profile and $\mathrm{C} \%$ in the foreign profile. Here $\mathrm{a} \%+\mathrm{b} \%=100 \%$ and $\mathrm{B} \%+\mathrm{C} \%=100 \%$.

Step 3: We calculate proportion $(b \%)$ of common species in the domestic profile, then scale to proportions $(\mathrm{B} \%)$ of common species in the foreign profile. At the same time, we scale 
the proportion of unique species in the domestic profile to be $\mathrm{A} \%(=\mathrm{a} \div \mathrm{b} \times \mathrm{B} \%)$. Because the foreign profile generally has more comprehensive species and the common species account for a smaller proportion. In order to include more species, we use the proportion of common species in the foreign profile as a reference for scaling.

Step 4: We integrate proportions of common species (B\%), unique species in the domestic profile $(\mathrm{A} \%)$ and unique species in the foreign profile $(\mathrm{C} \%)$ into a new and complete source profile. Finally, the proportions of unique species in the domestic profile, common species in both the domestic and foreign profiles, and unique species in the foreign profiles are $\mathrm{A} /(\mathrm{A}+\mathrm{B}+\mathrm{C}) \%, \mathrm{~B} /(\mathrm{A}+\mathrm{B}+\mathrm{C}) \%$, and $\mathrm{C} /(\mathrm{A}+\mathrm{B}+\mathrm{C}) \%$, respectively.

Figure S3-S12 show the procedure and data source of source profiles for architectural coating, furniture coating, automobile coating, other coating, offset printing ink, letterpress printing ink, gravure printing ink, other printing ink, shoemaking adhesive and herbicide. Here, we take the architectural coating as an example to elaborate the detailed procedure for the chemical speciation following the four-step procedure (Figure S3).

Firstly, the domestic profiles of Yuan et al. (2010) and Wang et al. (2014) are averaged to form a new domestic profile, while the foreign profile of McDonald et al. (2018) is used.

Secondly, the common species in the domestic profile and foreign profiles are identified, accounting for $88.8 \%(\mathrm{~b} \%)$ and $25.4 \%(\mathrm{~B} \%)$, respectively. The unique species account for $11.2 \%$ $(\mathrm{a} \%)$ in domestic profile while $74.6 \%(\mathrm{C} \%)$ in foreign profile.

Thirdly, the proportion of common species in domestic profile $(88.8 \%)$ is scaled to proportion in foreign profile $(25.4 \%$; $\mathrm{B} \%)$. The proportion of unique species in domestic profile is scaled to be $3.2 \%(=11.2 \% \div 88.8 \times 25.4 \% ; \mathrm{A} \%)$.

Finally, we normalized the proportions of common species, and unique species in the domestic and foreign profiles to generate the integrated profile. 
Table S1 Data source for VOC content of coating (\%).

\begin{tabular}{|c|c|c|c|c|c|c|c|c|c|c|}
\hline Produc & & Jiangsu & Shandong & Zhejiang & Guangdong & Shanghai & Beijing & GB18582-2008 & GB24408-2009 & Mean \\
\hline \multirow{5}{*}{ Architectural coating } & \multirow{3}{*}{ Solvent-based } & & & & & & & & 68 & \multirow{3}{*}{71} \\
\hline & & & & & & & & & 70 & \\
\hline & & & & & & & & & 76 & \\
\hline & \multirow{2}{*}{ Water-based } & & & & & & & 12 & 12 & \multirow{2}{*}{13} \\
\hline & & & & & & & & & 15 & \\
\hline \multirow{5}{*}{ Furniture coating } & \multirow{3}{*}{ Solvent-based } & 80 & 65 & 75 & 66 & & 60 & & & \multirow{3}{*}{69} \\
\hline & & & & 80 & 50 & & & & & \\
\hline & & & & & 75 & & & & & \\
\hline & Water-based & 15 & 10 & 30 & 14 & & 5 & & & 15 \\
\hline & UV & & 10 & & 14 & & & & & 12 \\
\hline \multirow{9}{*}{ Automotive coating } & \multirow{4}{*}{ Solvent-based } & 80 & 45 & 45 & 50 & 45 & & & & \multirow{4}{*}{61} \\
\hline & & & 80 & 80 & 45 & 80 & & & & \\
\hline & & & 55 & 55 & 80 & 55 & & & & \\
\hline & & & & & 55 & & & & & \\
\hline & \multirow{4}{*}{ Water-based } & 15 & & & 5 & & & & & \multirow{4}{*}{14} \\
\hline & & & & & 15 & & & & & \\
\hline & & & & & 15 & & & & & \\
\hline & & & & & 20 & & & & & \\
\hline & UV & & 10 & & 14 & & & & & 12 \\
\hline \multirow{3}{*}{ Powder coating } & & 0 & 0 & 2 & & & & & & \multirow{3}{*}{1} \\
\hline & & & 0 & & & & & & & \\
\hline & & & 5 & & & & & & & \\
\hline \multirow{5}{*}{ Other coating } & & 80 & 70 & 65 & 70 & 65 & & & & \multirow{3}{*}{67} \\
\hline & Solvent-based & & & 60 & 40 & & & & & \\
\hline & & & & 80 & 70 & & & & & \\
\hline & Water-based & 15 & 10 & 30 & & & & & & 18 \\
\hline & UV & & 10 & & 14 & & & & & 12 \\
\hline
\end{tabular}

a. GB18582-2008, Limit of harmful substances of interior architectural coatings; b. GB24408-2009, Limit of harmful substances of exterior wall coatings. 
Table S2 Data source for VOC content of ink (\%)

\begin{tabular}{|c|c|c|c|c|c|c|c|c|}
\hline & Products & Jiangsu & Zhejiang & Guangdong & Shanghai & $\mathrm{BJX}^{\mathrm{a}}$ & HJ $2542-2016^{b}$ & Mean \\
\hline \multirow{3}{*}{ Offset ink } & Sheet-fed offset Ink & 5 & 5 & 5 & 5 & 2 & 3 & 4 \\
\hline & Cold set web-fed-offset ink & & & 5 & & 2 & 3 & 3 \\
\hline & Heat set web-fed-offset ink & 30 & 30 & 30 & 30 & 5 & 10 & 23 \\
\hline \multirow{2}{*}{ Letterpress ink } & Solvent-based & 60 & 60 & 60 & 60 & 60 & & 60 \\
\hline & Water-based & 15 & & & & 10 & & 13 \\
\hline \multirow{2}{*}{ Gravure ink } & Solvent-based & 60 & 60 & 60 & 60 & 75 & & 63 \\
\hline & Water-based & 15 & & & & 30 & & 23 \\
\hline UV ink & & 0 & & & & 2 & & 1 \\
\hline Screen ink & & 45 & 45 & 45 & 45 & 50 & & 46 \\
\hline Other ink & & 60 & 60 & 60 & 60 & & & 60 \\
\hline
\end{tabular}

a. Data from VOCs.BJX (2019)

b. HJ 2542-2016, Technical requirement for environmental labelling products offset printing ink 
Table S3. Data source for VOC content of adhesive (\%)

Table S3a VOCs content of water-based adhesive

\begin{tabular}{|c|c|c|c|c|c|c|c|c|}
\hline \multirow{2}{*}{ Application fields } & \multicolumn{8}{|c|}{ Water-based (\%) ${ }^{\text {a }}$} \\
\hline & Polyvinyl acetate & Polyvinyl alcohol & Rubber & Polyurethane & EAV & Acrylics & Other & Mean \\
\hline Architecture & 10 & 10 & 15 & 10 & 5 & 10 & 5 & 9 \\
\hline Packaging and labelling & 5 & & 5 & 5 & 5 & 5 & 5 & 5 \\
\hline Woodworking & 10 & & 10 & 5 & 5 & 5 & 5 & 7 \\
\hline Paper converting & 5 & 5 & 5 & 5 & 5 & 5 & 5 & 5 \\
\hline Shoemaking & 5 & & 15 & 5 & 5 & 10 & 5 & 8 \\
\hline Fiber processing & 5 & 5 & 5 & 5 & 5 & 5 & 5 & 5 \\
\hline Transportation & 5 & & 5 & 5 & 5 & 5 & 5 & 5 \\
\hline Residential use & 5 & 5 & 5 & 5 & 5 & 5 & 5 & 5 \\
\hline Other & 5 & 5 & 5 & 5 & 5 & 5 & 5 & 5 \\
\hline
\end{tabular}

a. Data from GB33372-2020, Limit of volatile organic compounds content in adhesive.

Table S3b VOCs content of solvent-based adhesive

\begin{tabular}{|c|c|c|c|c|c|c|}
\hline \multirow{2}{*}{ Application fields } & \multicolumn{6}{|c|}{ Solvent-based (\%) ${ }^{\text {a }}$} \\
\hline & Polychloroprene rubber & SBS resin & Polyurethane & Acrylics & Other & Mean \\
\hline Architecture & 65 & 55 & 50 & 51 & 50 & 54 \\
\hline Packaging and labelling & 60 & 50 & 40 & 51 & 50 & 50 \\
\hline Woodworking & 60 & 50 & 40 & 51 & 40 & 48 \\
\hline Paper converting & 60 & 50 & 25 & 51 & 25 & 42 \\
\hline Shoemaking & 60 & 50 & 40 & & 40 & 48 \\
\hline Fiber processing & 60 & 50 & 25 & 51 & 25 & 42 \\
\hline Transportation & 60 & 50 & 25 & 51 & 25 & 42 \\
\hline Residential use & 60 & 50 & 25 & 51 & 25 & 42 \\
\hline Other & 60 & 50 & 25 & 51 & 25 & 42 \\
\hline
\end{tabular}

Table S3c VOCs content of bulk form adhesive

\begin{tabular}{|c|c|c|c|c|c|c|c|c|c|c|}
\hline \multirow{2}{*}{$\begin{array}{l}\text { Application } \\
\text { fields }\end{array}$} & \multicolumn{10}{|c|}{ Bulk form (\%) ${ }^{a}$} \\
\hline & Organic silicon & MS & Polyurethane & Polysulfide & Acrylics & Epoxy resins & $\alpha$-Cyanoacrylate & Thermoplastic & Other & Mean \\
\hline Architecture & 10 & 10 & 5 & 5 & & 10 & 2 & 5 & 5 & 7 \\
\hline $\begin{array}{c}\text { Packaging and } \\
\text { labelling }\end{array}$ & 10 & 5 & 5 & & & & & 5 & 5 & 6 \\
\hline Woodworking & 10 & 5 & 5 & 5 & 20 & 5 & 2 & 5 & 5 & 7 \\
\hline Paper converting & & 5 & 5 & & & & & 5 & 5 & 5 \\
\hline Shoemaking & & 5 & 5 & & & & 2 & 5 & 5 & 4 \\
\hline Fiber processing & & 5 & 5 & & & & & 5 & 5 & 5 \\
\hline Transportation & 10 & 10 & 5 & 5 & 20 & 10 & 2 & 5 & 5 & 8 \\
\hline Residential use & 10 & 5 & 5 & 5 & 20 & 5 & 2 & 5 & 5 & 7 \\
\hline Other & 10 & 5 & 5 & 5 & 20 & 5 & 2 & 5 & 5 & 7 \\
\hline
\end{tabular}


Table S4. Data source for VOC content of cleaner and insecticide (\%).

\begin{tabular}{|c|c|c|c|c|c|}
\hline & Products & & VOC limit (\%) & References & Mean \\
\hline \multirow{8}{*}{ Laundry } & \multirow{2}{*}{ Fabric refresher } & Aerosol & 15 & \multirow{8}{*}{ CARB } & \multirow{8}{*}{16} \\
\hline & & Nonaerosol & 6 & & \\
\hline & \multirow{2}{*}{ Fabric protectant } & Aerosol & 60 & & \\
\hline & & Nonaerosol & 1 & & \\
\hline & \multirow{2}{*}{ Laundry prewash } & Aerosol/Solid & 22 & & \\
\hline & & All other forms & 5 & & \\
\hline & \multirow{2}{*}{ Spot remover } & Aerosol & 15 & & \\
\hline & & Nonaerosol & 3 & & \\
\hline Dishwashing & & & 10 & HSECSM & 10 \\
\hline \multirow{10}{*}{ Surface cleaners } & \multirow{2}{*}{ Glass cleaner } & Aerosol & 10 & \multirow{9}{*}{ CARB } & \multirow{10}{*}{9} \\
\hline & & Nonaerosol & 3 & & \\
\hline & Oven or grill & $\begin{array}{l}\text { Aerosol/Pump } \\
\text { spray }\end{array}$ & 8 & & \\
\hline & cleaner & Liquid & 5 & & \\
\hline & \multirow{5}{*}{$\begin{array}{c}\text { Toilet or urinal } \\
\text { care product } \\
\text { Bathroom and tile } \\
\text { cleaner }\end{array}$} & Nonaerosol & 4 & & \\
\hline & & Aerosol & 10 & & \\
\hline & & Nonaerosol & 3 & & \\
\hline & & Aerosol & 7 & & \\
\hline & & Nonaerosol & 1 & & \\
\hline & Oil stain cleaner & & 40 & HSECSM & \\
\hline \multirow{2}{*}{ Industrial detergent } & & Water-based & 5 & \multirow{2}{*}{ GB 38508-2020 a } & 5 \\
\hline & & Solvent-based & 90 & & 90 \\
\hline \multirow{2}{*}{ Insecticide } & & Liquid & 25 & \multirow{2}{*}{ HSECSM } & \multirow{2}{*}{24} \\
\hline & & Spray & 22 & & \\
\hline
\end{tabular}

a. GB 38508-2020, Limits for volatile organic compounds content in cleaning agents 
Table S5. Data source for VOC content of personal care $(\%)$.

\begin{tabular}{|c|c|c|c|c|c|}
\hline & Products & & VOC limit (\%) & References & Mean \\
\hline \multirow{9}{*}{ Hair and body care } & Hair mousse & & 6 & \multirow{9}{*}{ CARB } & \multirow{9}{*}{23} \\
\hline & Hair shine & & 55 & & \\
\hline & Hair finishing spray & & 55 & & \\
\hline & Hair styling product & $\begin{array}{l}\text { Aerosol and } \\
\text { pump spray }\end{array}$ & 6 & & \\
\hline & & All other forms & 2 & & \\
\hline & Temporary hair color & & 55 & & \\
\hline & Hand cleaner & & 8 & & \\
\hline & Shampoo ${ }^{\text {a }}$ & & 8 & & \\
\hline & Body wash ${ }^{a}$ & & 8 & & \\
\hline \multirow{2}{*}{ Perfumes } & & $\begin{array}{l}\text { With } 20 \% \text { or less } \\
\text { fragrance }\end{array}$ & 75 & \multirow{2}{*}{ CARB } & \multirow{2}{*}{70} \\
\hline & & $\begin{array}{l}\text { With more than } \\
20 \% \text { fragrance }\end{array}$ & 65 & & \\
\hline Skin care & & & 35 & Green Seal & 35 \\
\hline \multirow{8}{*}{ Other cosmetic } & \multirow{2}{*}{ Antiperspirant } & Aerosol & 50 & \multirow{7}{*}{ CARB } & \multirow{8}{*}{18} \\
\hline & & Nonaerosol & 0 & & \\
\hline & \multirow{2}{*}{ Deodorant } & Aerosol & 10 & & \\
\hline & & Nonaerosol & 0 & & \\
\hline & Shaving cream & & 5 & & \\
\hline & Shaving gel & & 4 & & \\
\hline & Nail polish remover & & 1 & & \\
\hline & Nail polish & & 75 & Green Seal & \\
\hline
\end{tabular}

a. VOC content of shampoo and body wash is referred from data of hand cleaner.

Table S6. Five-tier evaluation system for uncertainty in activity data (Wei et al., 2011a).

\begin{tabular}{lll}
\hline Tier & Data source & Uncertainty \\
\cline { 2 - 3 } I & Directly from official statistics & $\pm 30 \%$ \\
II & $\begin{array}{l}\text { Estimated from other statistical information or reports; The data is } \\
\text { strongly related; The statistical information or reports is reliable. }\end{array}$ \\
III & $\begin{array}{l}\text { Estimated from other statistical information or reports; The data is } \\
\text { strongly related; The statistical information or reports are less reliable. }\end{array}$ \\
IV & $\begin{array}{l}\text { Estimated from other statistical information or reports; The data is less } \\
\text { related; The statistical information or reports is reliable. }\end{array}$ \\
V & $\begin{array}{l}\text { The data is less related; The statistical information or reports is less } \\
\text { reliable. }\end{array}$ \\
\end{tabular}


Table S7. The parameters for calculating emission of various solvent product.

\begin{tabular}{|c|c|c|c|c|c|c|c|c|}
\hline \multicolumn{3}{|c|}{ Products } & \multirow{2}{*}{$W_{V O C}(\%)$} & \multirow{2}{*}{$f_{V O C}$} & \multirow{2}{*}{$f_{S / I V O C}$} & \multirow{2}{*}{$W_{S / I V O C}(\%)$} & \multirow{2}{*}{$V F_{V O C}$} & \multirow{2}{*}{$V F_{S / I V O C}$} \\
\hline Level 1 & Level 2 & Level 3 & & & & & & \\
\hline \multirow{12}{*}{ Coating $^{a}$} & \multirow{2}{*}{$\begin{array}{l}\text { Architectural } \\
\text { coating }\end{array}$} & Solvent-based & $71 \pm 3$ & 0.99 & 0.01 & $1 \pm 0$ & 1 & $0.70 \pm 0.20$ \\
\hline & & Water-based & $13 \pm 1$ & 0.76 & 0.24 & $4 \pm 0$ & 1 & $0.70 \pm 0.20$ \\
\hline & \multirow{3}{*}{$\begin{array}{l}\text { Furniture } \\
\text { coating* }\end{array}$} & Solvent-based & $69 \pm 10$ & 0.99 & 0.01 & $1 \pm 0$ & 1 & $0.70 \pm 0.20$ \\
\hline & & Water-based & $15 \pm 8$ & 0.76 & 0.24 & $5 \pm 3$ & 1 & $0.70 \pm 0.20$ \\
\hline & & UV & $12 \pm 2$ & 0.76 & 0.24 & $4 \pm 1$ & 1 & $0.70 \pm 0.20$ \\
\hline & \multirow{3}{*}{$\begin{array}{l}\text { Automotive } \\
\text { coating* }\end{array}$} & Solvent-based & $61 \pm 15$ & 0.99 & 0.01 & $1 \pm 0$ & 1 & $0.70 \pm 0.20$ \\
\hline & & Water-based & $14 \pm 5$ & 0.76 & 0.24 & $4 \pm 1$ & 1 & $0.70 \pm 0.20$ \\
\hline & & UV & $12 \pm 2$ & 0.76 & 0.24 & $4 \pm 1$ & 1 & $0.70 \pm 0.20$ \\
\hline & Powder coating* & & $1 \pm 2$ & 0.76 & 0.24 & $0 \pm 0$ & 1 & $0.70 \pm 0.20$ \\
\hline & \multirow{3}{*}{ Other* } & Solvent-based & $67 \pm 11$ & 0.99 & 0.01 & $1 \pm 0$ & 1 & $0.70 \pm 0.20$ \\
\hline & & Water-based & $18 \pm 8$ & 0.76 & 0.24 & $6 \pm 3$ & 1 & $0.70 \pm 0.20$ \\
\hline & & UV & $12 \pm 2$ & 0.76 & 0.24 & $4 \pm 1$ & 1 & $0.70 \pm 0.20$ \\
\hline \multirow{10}{*}{$\operatorname{Ink}^{b}$} & \multirow{3}{*}{ Offset ink } & Sheet-fed offset Ink & $4 \pm 1$ & 0.86 & 0.14 & $1 \pm 0$ & 1 & $0.70 \pm 0.20$ \\
\hline & & Cold set web-fed-offset ink & $3 \pm 1$ & 0.86 & 0.14 & $0 \pm 0$ & 1 & $0.70 \pm 0.20$ \\
\hline & & Heat set web-fed-offset ink & $23 \pm 11$ & 0.86 & 0.14 & $4 \pm 2$ & 1 & $0.70 \pm 0.20$ \\
\hline & \multirow{2}{*}{ Letterpress ink } & Solvent-based & $60 \pm 0$ & 0.86 & 0.14 & $10 \pm 0$ & 1 & $0.70 \pm 0.20$ \\
\hline & & Water-based & $13 \pm 3$ & 0.86 & 0.14 & $2 \pm 0$ & 1 & $0.70 \pm 0.20$ \\
\hline & \multirow{2}{*}{ Gravure ink } & Solvent-based & $63 \pm 6$ & 0.86 & 0.14 & $10 \pm 1$ & 1 & $0.70 \pm 0.20$ \\
\hline & & Water-based & $23 \pm 8$ & 0.86 & 0.14 & $4 \pm 1$ & 1 & $0.70 \pm 0.20$ \\
\hline & UV ink & & $1 \pm 1$ & 0.86 & 0.14 & $0 \pm 0$ & 1 & $0.70 \pm 0.20$ \\
\hline & Screen ink & & $46 \pm 2$ & 0.86 & 0.14 & $7 \pm 0$ & 1 & $0.70 \pm 0.20$ \\
\hline & Other & & $60 \pm 0$ & 0.86 & 0.14 & $10 \pm 0$ & 1 & $0.70 \pm 0.20$ \\
\hline \multirow{2}{*}{ Adhesive $^{c}$} & \multirow{2}{*}{ Architecture } & Water-based & $9 \pm 3$ & 0.86 & 0.14 & $1 \pm 1$ & 1 & $0.70 \pm 0.20$ \\
\hline & & Solvent-based & $54 \pm 6$ & 0.89 & 0.11 & $7 \pm 1$ & 1 & $0.70 \pm 0.20$ \\
\hline
\end{tabular}




\begin{tabular}{|c|c|c|c|c|c|c|c|c|}
\hline \multicolumn{3}{|c|}{ Products } & \multirow{2}{*}{$W_{V O C}(\%)$} & \multirow{2}{*}{$f_{V O C}$} & \multirow{2}{*}{$f_{S / I V O C}$} & \multirow{2}{*}{$W_{S / I V O C}(\%)$} & \multirow{2}{*}{$V F_{V O C}$} & \multirow{2}{*}{$V F_{S / I V O C}$} \\
\hline Level 1 & Level 2 & Level 3 & & & & & & \\
\hline & & Bulk form & $7 \pm 3$ & 0.86 & 0.14 & $1 \pm 0$ & 1 & $0.70 \pm 0.20$ \\
\hline & \multirow{3}{*}{ Residential use } & Water-based & $5 \pm 0$ & 0.86 & 0.14 & $1 \pm 0$ & 1 & $0.70 \pm 0.20$ \\
\hline & & Solvent-based & $42 \pm 14$ & 0.89 & 0.11 & $5 \pm 2$ & 1 & $0.70 \pm 0.20$ \\
\hline & & Bulk form & $7 \pm 5$ & 0.86 & 0.14 & $1 \pm 1$ & 1 & $0.70 \pm 0.20$ \\
\hline & \multirow{4}{*}{ Woodworking* } & Water-based & $7 \pm 2$ & 0.86 & 0.14 & $1 \pm 0$ & 1 & $0.70 \pm 0.20$ \\
\hline & & Solvent-based & $48 \pm 8$ & 0.89 & 0.11 & $6 \pm 1$ & 1 & $0.70 \pm 0.20$ \\
\hline & & Bulk form & $7 \pm 5$ & 0.86 & 0.14 & $1 \pm 1$ & 1 & $0.70 \pm 0.20$ \\
\hline & & Formaldehyde type & $5 \pm 4^{d}$ & 0.86 & 0.14 & $1 \pm 1$ & 1 & $0.70 \pm 0.20$ \\
\hline & \multirow{3}{*}{$\begin{array}{c}\text { Paper } \\
\text { converting* }\end{array}$} & water-based & $5 \pm 0$ & 0.86 & 0.14 & $1 \pm 0$ & 1 & $0.70 \pm 0.20$ \\
\hline & & Solvent-based & $42 \pm 14$ & 0.89 & 0.11 & $5 \pm 2$ & 1 & $0.70 \pm 0.20$ \\
\hline & & Bulk form & $5 \pm 0$ & 0.86 & 0.14 & $1 \pm 0$ & 1 & $0.70 \pm 0.20$ \\
\hline & \multirow{3}{*}{ Shoemaking* } & Water-based & $8 \pm 4$ & 0.86 & 0.14 & $1 \pm 1$ & 1 & $0.70 \pm 0.20$ \\
\hline & & Solvent-based & $48 \pm 8$ & 0.89 & 0.11 & $6 \pm 1$ & 1 & $0.70 \pm 0.20$ \\
\hline & & Bulk form & $4 \pm 1$ & 0.86 & 0.14 & $1 \pm 0$ & 1 & $0.70 \pm 0.20$ \\
\hline & \multirow{3}{*}{$\begin{array}{c}\text { Fiber } \\
\text { processing* }\end{array}$} & Water-based & $5 \pm 0$ & 0.86 & 0.14 & $1 \pm 0$ & 1 & $0.70 \pm 0.20$ \\
\hline & & Solvent-based & $42 \pm 14$ & 0.89 & 0.11 & $5 \pm 2$ & 1 & $0.70 \pm 0.20$ \\
\hline & & Bulk form & $5 \pm 0$ & 0.86 & 0.14 & $1 \pm 0$ & 1 & $0.70 \pm 0.20$ \\
\hline & \multirow{3}{*}{ Transportation } & Water-based & $5 \pm 0$ & 0.86 & 0.14 & $1 \pm 0$ & 1 & $0.70 \pm 0.20$ \\
\hline & & Solvent-based & $42 \pm 14$ & 0.89 & 0.11 & $5 \pm 2$ & 1 & $0.70 \pm 0.20$ \\
\hline & & Bulk form & $8 \pm 5$ & 0.86 & 0.14 & $1 \pm 1$ & 1 & $0.70 \pm 0.20$ \\
\hline & \multirow{3}{*}{$\begin{array}{c}\text { Packaging and } \\
\text { labelling* }\end{array}$} & Water-based & $5 \pm 0$ & 0.86 & 0.14 & $1 \pm 0$ & 1 & $0.70 \pm 0.20$ \\
\hline & & Solvent-based & $50 \pm 6$ & 0.89 & 0.11 & $6 \pm 1$ & 1 & $0.70 \pm 0.20$ \\
\hline & & Bulk form & $6 \pm 2$ & 0.86 & 0.14 & $1 \pm 0$ & 1 & $0.70 \pm 0.20$ \\
\hline & \multirow{2}{*}{ Other } & Water-based & $5 \pm 0$ & 0.86 & 0.14 & $1 \pm 0$ & 1 & $0.70 \pm 0.20$ \\
\hline & & Solvent-based & $42 \pm 14$ & 0.89 & 0.11 & $5 \pm 2$ & 1 & $0.70 \pm 0.20$ \\
\hline
\end{tabular}




\begin{tabular}{|c|c|c|c|c|c|c|c|c|}
\hline \multicolumn{3}{|c|}{ Products } & \multirow{2}{*}{$W_{V O C}(\%)$} & \multirow{2}{*}{$f_{V O C}$} & \multirow{2}{*}{$f_{S / I V O C}$} & \multirow{2}{*}{$W_{S / I V O C}(\%)$} & \multirow{2}{*}{$V F_{V O C}$} & \multirow{2}{*}{$V F_{S / I V O C}$} \\
\hline Level 1 & Level 2 & Level 3 & & & & & & \\
\hline & & Bulk form & $7 \pm 5$ & 0.86 & 0.14 & $1 \pm 1$ & 1 & $0.70 \pm 0.20$ \\
\hline \multirow{3}{*}{ Pesticide } & Insecticide & & $24 \pm 2$ & 0.5 & 0.5 & $24 \pm 2$ & 1 & $0.30 \pm 0.25$ \\
\hline & Bactericide & & $24 \pm 2^{f}$ & 0.5 & 0.5 & $24 \pm 2$ & 1 & $0.30 \pm 0.25$ \\
\hline & Other & & $24 \pm 2^{f}$ & 0.5 & 0.5 & $24 \pm 2$ & 1 & $0.30 \pm 0.25$ \\
\hline \multirow[t]{3}{*}{ Cleaner } & Surface cleaner & & $9 \pm 11$ & 0.78 & 0.22 & $3 \pm 4$ & $0.47 \pm 0.38$ & 0 \\
\hline & \multirow{2}{*}{$\begin{array}{c}\text { Industrial } \\
\text { detergent } \mathrm{e}^{*}\end{array}$} & Water-based & $5 \pm 0$ & 0.22 & 0.78 & $18 \pm 0$ & 0.25 & 0.25 \\
\hline & & Solvent-based & $90 \pm 0$ & 0.99 & 0.01 & $1 \pm 0$ & 1 & $0.70 \pm 0.20$ \\
\hline Personal care & $\begin{array}{c}\text { Hair and body } \\
\text { care }\end{array}$ & & $23 \pm 23$ & 0.91 & 0.09 & $2 \pm 2$ & $0.60 \pm 0.40$ & 0 \\
\hline
\end{tabular}

a. The total of coating activity comes from China coating industry Yearbook. Level 2 of coating are derived from the China Coating Industry Yearbook for 2007-2010 and the "13th Five-Year plan" of China coating industry for 2011-2014. Level 3 of architectural coating is from Liu (2000) for 2000, China coating industry Yearbook for 2007-2010, Chyxx (2014) for 2013and Huaon (2019) for 2018. Years missing data are estimated based on the most recent years data available for level 2 and 3. Level 3 of other coating are estimated roughly based on water-based, solvent-based and UV proportion in total.

b. The total and level 2 of ink are obtained from China Light Industry Yearbook. Level 3 proportion for some ink are from Chyxx (2017), Bai and Zi (2003) and Qianzhan (2019).

c. The total and level 2 sources of adhesive are obtained from China Chemical Industry Yearbook. Solvent-based proportion of shoemaking adhesive is from Yuan and Chen (2005) and Chyxx (2015). Other level 3 are estimated roughly based on water-based, solvent-based and bulk form proportion in total.

d. VOCs content of formaldehyde type adhesive is referred to DYQ (2019), Henan River Grinding Material Co LTD (2020) and GB/T14732-2017 (Wood adhesive: urea-formaldehyde, phenol-formaldehyde and melamine-formaldehyde resins). e. Level 3 of industrial detergent is from Vzkoo (2019).

f. Data are referred to that of insecticide.

* indicates the industrial solvent use. 
Table S8. Proxy variables for allocating national emissions to provincial level.

\begin{tabular}{|c|c|c|}
\hline \multicolumn{2}{|r|}{ Solvent use } & Proxy variable \\
\hline \multicolumn{2}{|r|}{ Pesticide } & Cultivated land area ${ }^{a}$ \\
\hline \multicolumn{2}{|r|}{ Personal care } & Disposable income of households ${ }^{\text {a }}$ \\
\hline \multirow{2}{*}{ Cleaner } & Industrial detergent & Industrial sales value ${ }^{b}$ \\
\hline & Other cleaner & Disposable income of households ${ }^{\text {a }}$ \\
\hline \multirow{4}{*}{ Coating } & Architectural coating & Building area completed ${ }^{\text {a }}$ \\
\hline & Automotive coatings & Sales value of automobile manufacturing ${ }^{b}$ \\
\hline & Furniture coatings & Sales value of furniture manufacturing ${ }^{b}$ \\
\hline & Other coatings & Industrial sales value ${ }^{b}$ \\
\hline \multicolumn{2}{|r|}{ Ink } & $\begin{array}{l}\text { Sales value of printing and recording media } \\
\text { reproduction industry }{ }^{b}\end{array}$ \\
\hline \multirow{8}{*}{ Adhesive } & adhesives for wood processing & Sales value of wood processing ${ }^{b}$ \\
\hline & Adhesives for paper processing, label & $\begin{array}{c}\text { Sales value of printing and recording media } \\
\text { reproduction industry }{ }^{\mathrm{b}}\end{array}$ \\
\hline & Adhesives for fiber processing & Sales value of fiber processing ${ }^{b}$ \\
\hline & Adhesives for transportation & Sales value of automobile manufacturing ${ }^{b}$ \\
\hline & Adhesives for building & Building area completed ${ }^{\text {a }}$ \\
\hline & Adhesives for making shoes & Sales value of making shoes ${ }^{b}$ \\
\hline & Adhesives for residential use & Disposable income of households ${ }^{a}$ \\
\hline & Other adhesives & Industrial sales value ${ }^{b}$ \\
\hline
\end{tabular}

a. Data from 2017 China Statistical Yearbook

b. Data from 2017 China Industry Statistical Yearbook 
Table S9. List of VOCs and S/IVOCs and their MIR and SOA yield values used in this study.

\begin{tabular}{|c|c|c|c|}
\hline No. & Species & MIR & SOA yield \\
\hline \multicolumn{4}{|c|}{ Alkanes } \\
\hline 1 & Ethane & 0.28 & 0 \\
\hline 2 & Propane & 0.49 & 0 \\
\hline 3 & Isobutane & 1.23 & 0 \\
\hline 4 & N-butane & 1.15 & 0 \\
\hline 5 & Cyclopentane & 2.39 & 0.012 \\
\hline 6 & Isopentane & 1.45 & 0 \\
\hline 7 & N-pentane & 1.31 & 0 \\
\hline 8 & methylcyclopentane & 2.19 & 0.022 \\
\hline 9 & Cyclohexane & 1.25 & 0.022 \\
\hline 10 & 2,2-dimethylbutane & 1.17 & 0 \\
\hline 11 & 2,3-dimethylbutane & 0.97 & 0 \\
\hline 12 & 2-methylpentane & 1.5 & 0 \\
\hline 13 & 3-methylpentane & 1.8 & 0 \\
\hline 14 & N-hexane & 1.24 & 0.0028 \\
\hline 15 & Methylcyclohexane & 1.7 & 0.035 \\
\hline 16 & 2,3-dimethylpentane & 1.34 & 0.0083 \\
\hline 17 & 2,4-dimethylpentane & 1.55 & 0.0083 \\
\hline 18 & 2-methylhexane & 1.19 & 0 \\
\hline 19 & 3-methylhexane & 1.61 & 0 \\
\hline 20 & N-heptane & 1.07 & 0.0066 \\
\hline 21 & Ethylcyclohexane & 1.47 & 0.051 \\
\hline 22 & 2,2,4-Trimethylpentane & 1.26 & 0.003 \\
\hline 23 & 2,3,4-Trimethylpentane & 1.03 & 0.003 \\
\hline 24 & 2-methylheptane & 1.07 & 0.003 \\
\hline 25 & 3-methylheptane & 1.24 & 0.003 \\
\hline 26 & N-octane & 0.9 & 0.013 \\
\hline 27 & 1,2-Dimethylcyclohexane & 1.41 & 0.051 \\
\hline 28 & 1,3-Dimethylcyclohexane & 1.52 & 0.051 \\
\hline 29 & 4-Methylheptane & 1.25 & 0.003 \\
\hline 30 & N-nonane & 0.78 & 0.021 \\
\hline 31 & 1,1,3-Trimethylcyclohexane & 1.19 & 0.068 \\
\hline 32 & 1,2,4-Trimethylcyclohexane & 1.36 & 0.068 \\
\hline 33 & 1-Ethyl-3-methylcyclohexane & 1.36 & 0.068 \\
\hline 34 & 1-Ethyl-4-methylcyclohexane & 1.44 & 0.068 \\
\hline 35 & 2,5-Dimethylheptane & 1.35 & 0.0083 \\
\hline 36 & 3-Ethylheptane & 1.1 & 0.0083 \\
\hline 37 & 3-Methyloctane & 0.99 & 0.0083 \\
\hline 38 & 4-Methyloctane & 0.95 & 0.0083 \\
\hline 39 & N-decane & 0.68 & 0.033 \\
\hline 40 & 2,6-Dimethyloctane & 1.08 & 0.02 \\
\hline
\end{tabular}


Table S9 (continued).

\begin{tabular}{|c|c|c|c|}
\hline No. & Species & MIR & SOA yield \\
\hline 41 & 2-Methylnonane & 0.73 & 0.02 \\
\hline 42 & Endo-tricyclo[5.2.1.0(2.6)]decane & 1.09 & 0.068 \\
\hline 43 & N-undecane & 0.61 & 0.05 \\
\hline 44 & N-dodecane & 0.55 & 0.069 \\
\hline 45 & 2,2,4,6,6-Pentamethylheptane & 0.63 & 0.069 \\
\hline 46 & n-tetradecane & 0.51 & 0.11 \\
\hline 47 & n-pentadecane & 0.5 & 0.14 \\
\hline 48 & n-Hexadecane & 0.45 & 0.19 \\
\hline 49 & n-Heptadecane & 0.42 & 0.26 \\
\hline 50 & N-tridecane & 0.53 & 0.09 \\
\hline 51 & branched C9 alkanes & 1.14 & 0.0083 \\
\hline 52 & branched $\mathrm{C} 10$ alkanes & 0.94 & 0.02 \\
\hline 53 & branched C11 alkanes & 0.73 & 0.04 \\
\hline 54 & branched $\mathrm{C} 8$ alkanes & 1.45 & 0.003 \\
\hline 55 & branched C6 alkanes & 1.31 & 0 \\
\hline 56 & branched C7 alkanes & 1.48 & 0 \\
\hline 57 & branched C5 alkanes & 1.45 & 0 \\
\hline 58 & branched $\mathrm{C} 12$ alkanes & 0.63 & 0.069 \\
\hline 59 & branched $\mathrm{C} 13$ alkanes & 0.6 & 0.11 \\
\hline 60 & branched $\mathrm{C} 16$ alkanes & 0.47 & 0.3 \\
\hline 61 & branched $\mathrm{C} 15$ alkanes & 0.5 & 0.22 \\
\hline 62 & branched $\mathrm{C} 17$ alkanes & 0.44 & 0.41 \\
\hline 63 & branched C14 alkanes & 0.55 & 0.15 \\
\hline 64 & $2,2,4,4,6,8,8$-heptamethylnonane & 0.47 & 0.3 \\
\hline 65 & C8 cycloalkanes & 1.47 & 0.051 \\
\hline 66 & C9 cycloalkanes & 1.36 & 0.068 \\
\hline 67 & C11 cycloalkanes & 0.9 & 0.11 \\
\hline 68 & C10 cycloalkanes & 1.07 & 0.087 \\
\hline 69 & C7 cycloalkanes & 1.7 & 0.035 \\
\hline 70 & C6 cycloalkanes & 1.25 & 0.022 \\
\hline 71 & C10 bicycloalkanes & 1.09 & 0.087 \\
\hline 72 & C11 bicycloalkanes & 0.91 & 0.11 \\
\hline 73 & C12 cycloalkanes & 0.8 & 0.15 \\
\hline 74 & C15 cycloalkanes & 0.61 & 0.31 \\
\hline 75 & C16 cycloalkanes & 0.55 & 0.36 \\
\hline 76 & C14 cycloalkanes & 0.65 & 0.25 \\
\hline 77 & C13 cycloalkanes & 0.7 & 0.19 \\
\hline 78 & C17 cycloalkanes & 0.52 & 0.43 \\
\hline \multicolumn{4}{|c|}{ Alkenes } \\
\hline 79 & Ethylene & 9 & 0 \\
\hline 80 & Propylene & 11.66 & 0 \\
\hline
\end{tabular}


Table S9 (continued).

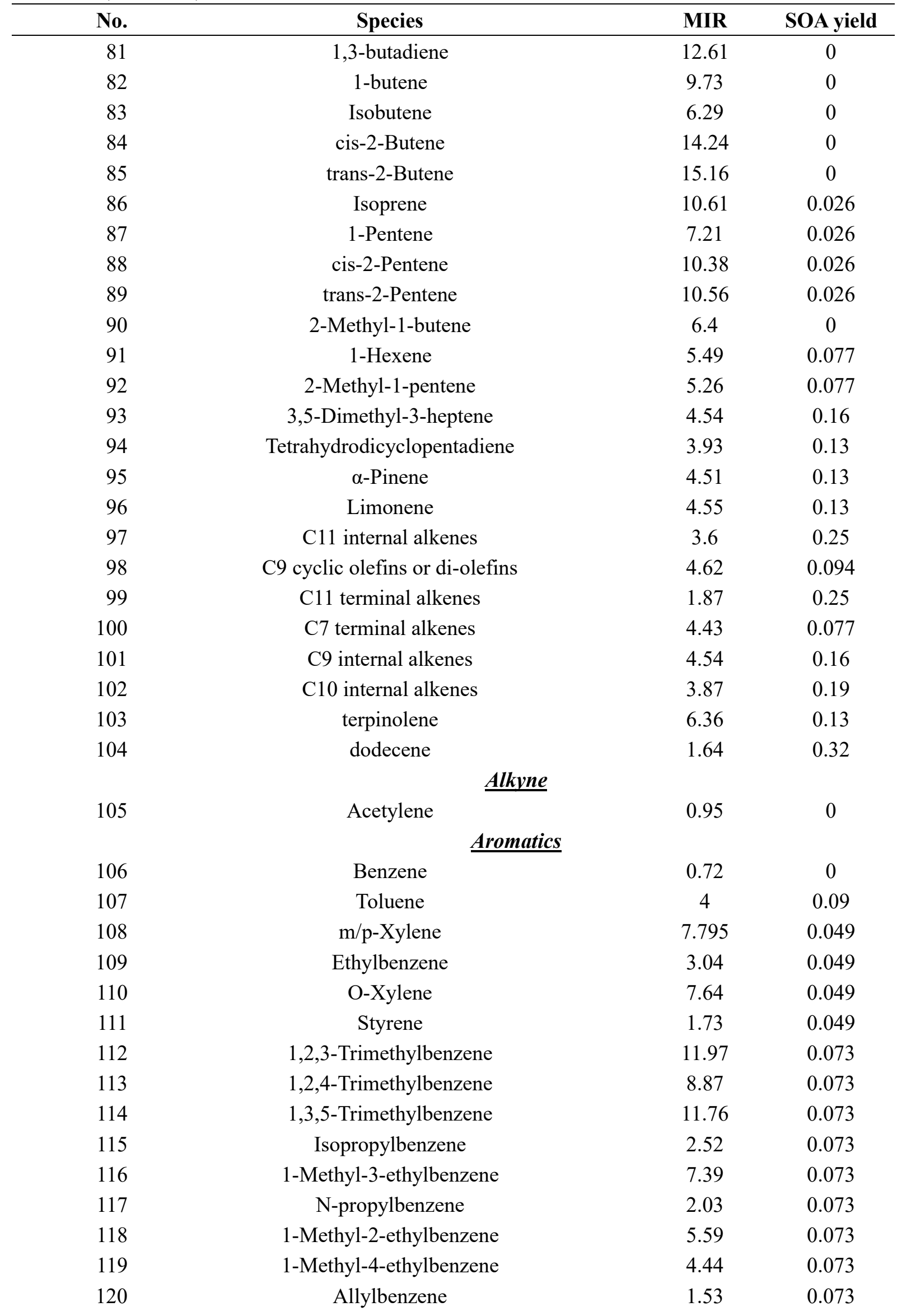


Cyclopropylbenzene indene

1,3-diethylbenzene

1,4-diethylbenzene

1,2-diethylbenzene

Naphthalene

2-Isopropyltoluene

4-Isopropyltoluene

1,2,3,4-tetramethylbenzene

1,2,3,5-tetramethylbenzene

1,2,4,5-tetramethylbenzene

1-Ethyl-2,4-dimethylbenzene

1-Methyl-3-Propylbenzene

1-Methyl-4-Propylbenzene

2-Ethyl-1,3-dimethylbenzene

2-Ethyl-1,4-dimethylbenzene

4-Ethyl-1,2-dimethylbenzene

1-Ethyl-2,3-dimethylbenzene sec-Butylbenzene p-sec-Butyltoluene

C10 disubstituted benzenes

C10 trisubstituted benzenes

C9 trisubstituted benzenes

C10 tetrasubstituted benzenes

$$
\text { Methyl indanes }
$$

C10 monosubstituted benzenes

C9 disubstituted benzenes

N-butylbenzene

C11 trisubstituted benzenes

C12 trisubstituted benzenes

C11 tetrasubstituted benzenes

C14 naphthalenes

C12 naphthalenes

C11 tetralins or indanes

C15 naphthalenes

C11 disubstituted benzenes

C13 naphthalenes

C13 trisubstituted benzenes

C14 trisubstituted benzenes

C11 monosubstituted benzenes

C11 pentasubstituted benzenes

C16 naphthalenes

2-Methylnaphthalene
2.03

0.073

1.55

0.16

7.1

0.1

4.43

0.1

5.49

0.1

3.34

0.2

5.49

0.1

4.44

0.1

9.26

0.1

9.26

0.1

9.26

0.1

7.55

0.1

7.1

0.1

4.43

0.1

10.15

0.1

7.55

0.1

7.55

0.1

10.15

0.1

2.36

0.1

4.92

0.15

5.68

0.1

9.26

0.1

10.87

0.073

9.26

0.1

2.97

0.21

2.36

0.1

5.81

0.073

2.36

0.1

8.13

0.15

7.3

0.23

8.13

0.15

3.3

0.49

3.89

0.36

2.69

0.28

3.06

0.52

4.92

0.15

3.57

0.43

6.57

0.34

6

0.46

2.12

0.15

$8.13 \quad 0.15$

$2.86 \quad 0.54$

3.06

\section{Oxygenated VOCS}




\begin{tabular}{|c|c|c|c|}
\hline 164 & Formaldehyde & 9.46 & 0 \\
\hline 165 & Ethanol & 1.53 & 0 \\
\hline 166 & Acraldehyde & 7.45 & 0 \\
\hline 167 & Acetone & 0.36 & 0 \\
\hline 168 & Isopropyl alcohol & 0.61 & 0 \\
\hline 169 & Methyl acetate & 0.072 & 0 \\
\hline 170 & Dimethoxymethane & 0.94 & 0 \\
\hline 171 & Dimethyl carbonate & 0.059 & 0 \\
\hline 172 & Vinyl acetate & 3.2 & 0 \\
\hline 173 & 2-Butanone & 1.48 & 0 \\
\hline 174 & Ethyl acetate & 0.63 & 0 \\
\hline 175 & Tetrahydrofuran & 4.31 & 0 \\
\hline 176 & n-butyl alcohol & 2.88 & 0 \\
\hline 177 & 2-Ethoxyethanol & 3.71 & 0 \\
\hline 178 & propylene glycol methyl ether & 2.44 & 0 \\
\hline 179 & tert-Butanol & 0.41 & 0 \\
\hline 180 & 2-Methoxy-2-propanol & 3.01 & 0 \\
\hline 181 & Methyl tert-butyl ether & 0.73 & 0.039 \\
\hline 182 & Methyl methacrylate & 15.61 & 0.0085 \\
\hline 183 & Ethyl Acrylate & 7.77 & 0.0085 \\
\hline 184 & Methyl isopropyl ether & 3.74 & 0 \\
\hline 185 & Propyl acetate & 0.78 & 0.0085 \\
\hline 186 & Methyl isobutyl ketone & 3.88 & 0.013 \\
\hline 187 & 2-hexanone & 3.14 & 0.013 \\
\hline 188 & N-butyl acetate & 0.83 & 0.014 \\
\hline 189 & Cyclohexanone & 1.35 & 0.0019 \\
\hline 190 & sec-Butyl acetate & 1.32 & 0.014 \\
\hline 191 & propylene glycol methyl ether acetate & 1.7 & 0.029 \\
\hline 192 & Isobutyl acetate & 0.62 & 0.014 \\
\hline 193 & 2-Ethoxyethyl acetate & 1.84 & 0.11 \\
\hline 194 & 2-Butoxyethanol & 2.9 & 0.052 \\
\hline 195 & Butyl Acrylate & 5.02 & 0.0215 \\
\hline 196 & 2-Pentyl Acetate & 1.33 & 0.0215 \\
\hline 197 & 2-Ethyl-1-hexanol & 2 & 0.16 \\
\hline 198 & 4-Methylbenzaldehyde & 3.16 & 0.002 \\
\hline 199 & 2,6-Dimethyl-4-heptanone & 2.68 & 0.029 \\
\hline 200 & 2-Phenyl-2-propanol & 4.53 & 0.036 \\
\hline 201 & Dimethyl ether & 0.81 & 0 \\
\hline 202 & methanol & 0.67 & 0 \\
\hline 203 & Ethanolamine & 6.81 & 0 \\
\hline 204 & 2-amino-2-methyl-1-propanol & 0.25 & 0 \\
\hline 205 & 1-Methyl-2-pyrrolidinone & 2.41 & 0.0019 \\
\hline 206 & Isopropyl acetate & 1.07 & 0.0085 \\
\hline 207 & Methyl amyl ketone & 1.48 & 0.014 \\
\hline
\end{tabular}




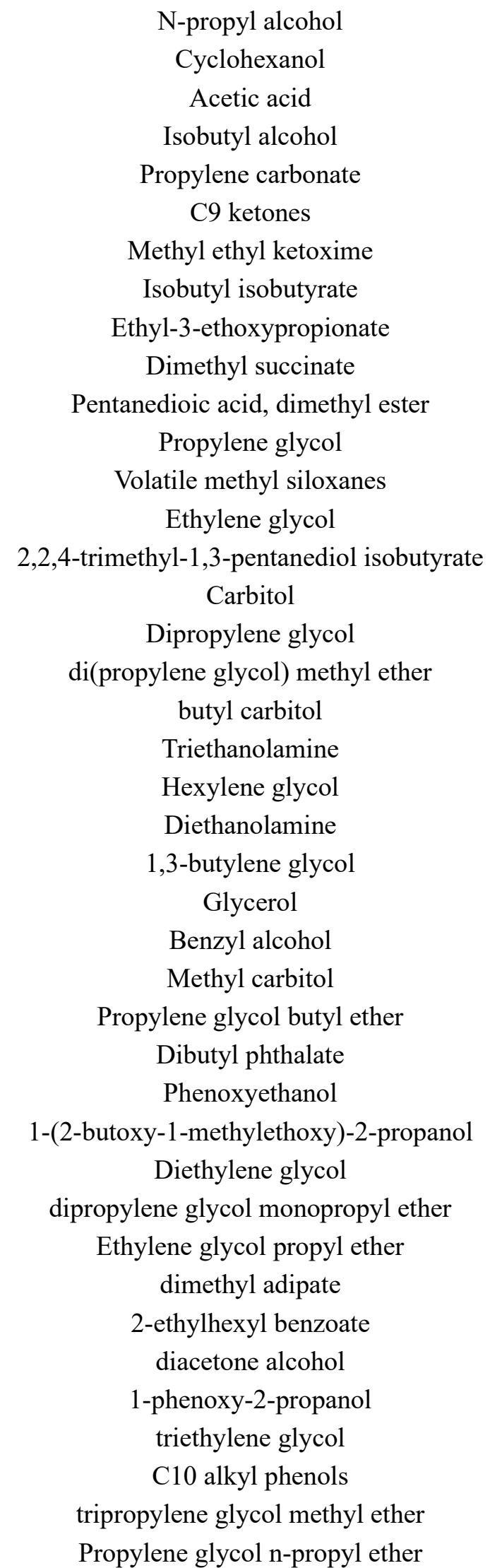

Halocarbons

$0 \quad 0$

$0.038 \quad 0$


Note: $\mathrm{C} \geq 12$ species are considered as S/IVOCs in this study.

\begin{tabular}{ccc} 
trichlorofluoromethane & 0 & 0 \\
Dichloromethane & 0.041 & 0 \\
Trichloromethane & 0.022 & 0 \\
Carbon tetrachloride & 0 & 0 \\
trans-1,2-Dichloroethylene & 1.7 & 0 \\
1,1-Dichloroethane & 0.069 & 0 \\
1,2-Dichloroethane & 0.21 & 0 \\
Trichloroethylene & 0.64 & 0 \\
1,1,2-Trichloroethane & 0.086 & 0 \\
Perchloroethylene & 0.031 & 0 \\
1,2-Dichloroacetylene & 0.17 & 0 \\
1,2-Dichloropropane & 0.29 & 0 \\
1,3-Dichloro-1-propene & 4.365 & 0 \\
Hexachlorobutadiene & 0.043 & 0 \\
Chlorobenzene & 0.32 & 0 \\
1,3-Dichlorobenzene & 0.178 & 0 \\
1,4-Dichlorobenzene & 0.178 & 0 \\
1,2-Dichlorobenzene & 0.178 & 0 \\
1,2,4-trichlorobenzene & 0.178 & 0 \\
Benzyl chloride & 4 & 0.09 \\
P-chlorobenzotrifluoride & 0.126 & 0 \\
Carbon disulfide & 0.25 & 0 \\
1,1-Difluoroethane & 0.0175 & 0 \\
1,1-dichloro-1-fluoroethane & 0.086 & 0 \\
Unspeciated (n-tetradecane) & 0 & 0.11 \\
\hline
\end{tabular}


Table S10 (a) Market share of NMVOCs control technologies and their control efficiency in the Yangtze River Delta (YRD) region (Lu et al., 2018).

\begin{tabular}{ccc}
\hline VOCs control technology & Share $(\%)$ & Control efficiency (\%) \\
\hline Adsorption & 48.8 & 45 \\
Absorption & 19.5 & 20 \\
Plasma & 10.3 & 30 \\
Direct catalytic combustion & 7.1 & 70 \\
Adsorption - Catalytic combustion & 6.3 & 63 \\
Combustion & 3.7 & 78 \\
Photolysis & 1.9 & 15 \\
Other technologies & 2.5 & $43^{\mathrm{a}}$ \\
\hline
\end{tabular}

Table S10 (b): Market share of NMVOCs control technologies and their control efficiency in the Pearl River Delta (PRD) region, China (Cai, 2016)

\begin{tabular}{ccc}
\hline VOCs control technology & Share $(\%)$ & Control efficiency $(\%)$ \\
\hline Adsorption $^{\text {Combination technologies }}{ }^{\mathrm{c}}$ & 68 & $45^{\mathrm{b}}$ \\
Absorption $^{\mathrm{d}}$ & 12 & $63^{\mathrm{d}}$ \\
Combustion $^{\mathrm{b}}$ & 11 & $20^{\mathrm{b}}$ \\
Plasma & 2 & $78^{\mathrm{b}}$ \\
Photolysis & 2 & $30^{\mathrm{b}}$ \\
Other technologies & 1 & $15^{\mathrm{b}}$ \\
\hline
\end{tabular}

\footnotetext{
a. Control efficiency of other technologies is based on mean of control efficiency of single technology in Table S8 (a)

b. Data are referred to Table S8 (a).

c. Combination technologies means that more than two technologies work together to process VOCs

d. Data is referred to that of adsorption - catalytic combustion in Table S8 (a).
} 
Table S11. Annual value of production for organic exhaust gas treatment industry, percentage with NMVOCs treatment facilities and effective control efficiency during 2000-2017.

\begin{tabular}{cccc}
\hline Year & $\begin{array}{c}\text { Annual value of production } \\
\text { (Billion CNY) }\end{array}$ & $\begin{array}{c}\text { Percentage with treatment } \\
\text { facilities }(\%)\end{array}$ & $\begin{array}{c}\text { Overall effective control } \\
\text { efficiency }(\%)\end{array}$ \\
\hline 2000 & 0.49 & 0 & 0 \\
2001 & 0.56 & 1 & 0.4 \\
2002 & 0.64 & 2 & 0.9 \\
2003 & 0.74 & 3 & 1.3 \\
2004 & 0.85 & 4 & 1.7 \\
2005 & 0.98 & 5 & 2.2 \\
2006 & 1.13 & 6 & 2.6 \\
2007 & 1.3 & 7 & 3.0 \\
2008 & 1.5 & 8 & 3.4 \\
2009 & 1.75 & 9 & 3.9 \\
2010 & 2.1 & 10 & 4.3 \\
2011 & 2.8 & 13.3 & 5.7 \\
2012 & 3.2 & 16.7 & 7.2 \\
2013 & 3.5 & 20 & 8.6 \\
2014 & 8.35 & 35 & 15.1 \\
2015 & 21.2 & 50 & 21.5 \\
2016 & 27 & 60 & 25.8 \\
2017 & 54 & 70 & 30.1 \\
\hline
\end{tabular}


Table S12. Solvent use emissions from various categories in 2000-2017 (Tg).

\begin{tabular}{cccccccccc}
\hline Year & Adhesive & Coating & Ink & Personal Care & Pesticide & Cleaner & Total & Lower limit & Upper limit \\
\hline 2000 & 0.30 & 0.80 & 0.06 & 0.35 & 0.05 & 0.01 & 1.58 & 1.19 & 2.22 \\
2001 & 0.32 & 0.76 & 0.07 & 0.44 & 0.06 & 0.01 & 1.66 & 1.24 & 2.38 \\
2002 & 0.38 & 0.81 & 0.08 & 0.49 & 0.07 & 0.01 & 1.85 & 1.38 & 2.68 \\
2003 & 0.44 & 0.91 & 0.08 & 0.66 & 0.09 & 0.02 & 2.19 & 1.60 & 3.32 \\
2004 & 0.47 & 1.10 & 0.09 & 0.70 & 0.16 & 0.02 & 2.53 & 1.89 & 3.72 \\
2005 & 0.52 & 1.47 & 0.09 & 0.75 & 0.21 & 0.02 & 3.06 & 2.31 & 4.43 \\
2006 & 0.65 & 1.81 & 0.10 & 0.77 & 0.30 & 0.02 & 3.65 & 2.79 & 5.14 \\
2007 & 0.78 & 2.54 & 0.11 & 0.87 & 0.40 & 0.01 & 4.71 & 3.57 & 6.56 \\
2008 & 0.83 & 2.73 & 0.12 & 0.99 & 0.46 & 0.02 & 5.14 & 3.88 & 7.17 \\
2009 & 0.97 & 3.06 & 0.16 & 1.04 & 0.56 & 0.03 & 5.82 & 4.47 & 7.90 \\
2010 & 1.20 & 3.92 & 0.16 & 1.09 & 0.55 & 0.03 & 6.96 & 5.28 & 9.41 \\
2011 & 1.40 & 4.29 & 0.18 & 1.26 & 0.59 & 0.04 & 7.76 & 5.88 & 10.61 \\
2012 & 1.49 & 4.96 & 0.19 & 1.47 & 0.85 & 0.04 & 9.00 & 6.76 & 12.60 \\
2013 & 1.63 & 4.97 & 0.20 & 1.68 & 0.68 & 0.04 & 9.19 & 6.83 & 13.06 \\
2014 & 1.65 & 6.13 & 0.19 & 1.77 & 0.84 & 0.06 & 10.64 & 7.82 & 14.96 \\
2015 & 1.62 & 5.77 & 0.18 & 1.87 & 0.83 & 0.07 & 10.34 & 7.65 & 14.57 \\
2016 & 1.65 & 5.77 & 0.18 & 2.10 & 0.77 & 0.07 & 10.53 & 7.72 & 14.84 \\
2017 & 1.63 & 6.10 & 0.18 & 2.15 & 0.43 & 0.06 & 10.55 & 7.73 & 14.94 \\
\hline
\end{tabular}


Table S13. Source categories, emission factors and activity data source for solvent use NMVOC emissions in different studies.

\begin{tabular}{|c|c|c|c|c|c|c|}
\hline Reference & Base year and & & mission sources & Emission factors & Units & Activity data sources or \\
\hline \multirow{14}{*}{ Bo et al. (2008) } & \multirow{14}{*}{ 2005, China } & \multirow{13}{*}{ Coating } & Architecture surface coating & 0.051 & $\mathrm{~kg} /$ capita & \multirow{14}{*}{$\begin{array}{l}\text { China Statistical Yearbooks, } \\
\text { China Light Industry } \\
\text { Yearbooks, China Market } \\
\text { Yearbooks, China Industrial } \\
\text { Economic Statistical Year }\end{array}$} \\
\hline & & & Can coating & 100 & $\mathrm{Mg} /$ wire & \\
\hline & & & Magnet wire coating & 84 & $\mathrm{Mg} /$ wire & \\
\hline & & & $\begin{array}{l}\text { Agriculture machines surface } \\
\text { coating }\end{array}$ & 236 & $\mathrm{Mg} /$ plant & \\
\hline & & & $\begin{array}{l}\text { Surface coating of plastic parts } \\
\text { for business machines }\end{array}$ & 236 & $\mathrm{Mg} /$ plant & \\
\hline & & & Metal furniture surface coating & 218 & $\mathrm{Mg} /$ plant & \\
\hline & & & Wood furniture & 0.4 & $\mathrm{~kg} /$ piece & \\
\hline & & & Machine tool equipment & 0.4 & $\mathrm{~kg} /$ piece & \\
\hline & & & $\begin{array}{c}\text { Automobile \& light duty truck } \\
\text { surface coating }\end{array}$ & 21.2 & $\mathrm{~kg} /$ vehicle & \\
\hline & & & Large appliance surface coating & 0.2 & $\mathrm{~kg} /$ production & \\
\hline & & & Bicycle surface coating & 0.3 & $\mathrm{~kg} / \mathrm{bike}$ & \\
\hline & & & Automobile recoating & 0.021 & $\mathrm{~kg} /$ capita & \\
\hline & & & Solvent degreasing & 0.044 & $\mathrm{~kg} /$ capita & \\
\hline & & Others & $\begin{array}{l}\text { Commercial/ Consumer solvent } \\
\text { use }\end{array}$ & 0.1 & $\mathrm{~kg} /$ capita & \\
\hline \multirow{6}{*}{ Wei et al. (2011b) } & \multirow{6}{*}{ 2010, China } & \multirow{6}{*}{ Coating } & Interior wall paint & 180 & $\mathrm{~g} / \mathrm{kg}$ paint & 2.2 \\
\hline & & & Exterior wall paint & 580 & $\mathrm{~g} / \mathrm{kg}$ paint & 1.7 \\
\hline & & & Auto-manufacturing paint & 470 & $\mathrm{~g} / \mathrm{kg}$ paint & 0.2 \\
\hline & & & Auto-repair paint & 720 & $\mathrm{~g} / \mathrm{kg}$ paint & 0.06 \\
\hline & & & Wood paint & 640 & $\mathrm{~g} / \mathrm{kg}$ paint & 1.3 \\
\hline & & & Other industrial paint & 375 & $\mathrm{~g} / \mathrm{kg}$ paint & 2.5 \\
\hline
\end{tabular}




\begin{tabular}{|c|c|c|c|c|c|c|}
\hline Reference & Base year and & \multicolumn{2}{|c|}{ Emission sources } & Emission factors & Units & Activity data sources or \\
\hline & & \multirow{2}{*}{ Adhesive } & Shoemaking adhesive & 670 & $\mathrm{~g} / \mathrm{kg}$ adhesive & 0.3 \\
\hline & & & Other adhesive & 90 & $\mathrm{~g} / \mathrm{kg}$ adhesive & 6.4 \\
\hline & & Ink & Printing & 1210 & $\mathrm{~g} / \mathrm{kg}$ ink & 0.45 \\
\hline \multirow{15}{*}{ Yin et al. (2015) } & \multirow{15}{*}{ 2010, the PRD } & \multirow{10}{*}{ Coating } & Architecture surface coating & 548.43 & $\mathrm{~g} / \mathrm{kg}$ & $\begin{array}{c}\text { Guangdong Statistical } \\
\text { Yearbook } 2011\end{array}$ \\
\hline & & & Car & 4.8 & $\mathrm{~kg} /$ per vehicle & \multirow{5}{*}{$\begin{array}{c}\text { China Automotive Industry } \\
\text { Yearbook } 2011\end{array}$} \\
\hline & & & Truck & 32 & $\mathrm{~kg} /$ per vehicle & \\
\hline & & & Passenger car & 7.8 & $\mathrm{~kg} /$ per vehicle & \\
\hline & & & Motorcycle & 1.5 & $\mathrm{~kg} /$ per vehicle & \\
\hline & & & Bicycle & 0.3 & $\mathrm{~kg} /$ per vehicle & \\
\hline & & & Shipping coating & 750 & $\mathrm{~g} / \mathrm{kg}$ & $\begin{array}{c}\text { China Machinery Industry } \\
\text { Yearbook } 2011\end{array}$ \\
\hline & & & Container coating & 750 & $\mathrm{~g} / \mathrm{kg}$ & Statistical Yearbook for \\
\hline & & & Furniture Surface coating & 0.54 & $\mathrm{~kg} /$ piece & each city in PRD \\
\hline & & & Toy manufacturing & 730 & $\mathrm{~kg} / \mathrm{t}$ & $\begin{array}{c}\text { Guangdong Statistical } \\
\text { Yearbook }\end{array}$ \\
\hline & & \multirow{2}{*}{ Adhesive } & Shoemaking & 0.06 & $\mathrm{~kg} /$ pair & \multirow{2}{*}{$\begin{array}{l}\text { Statistical Yearbook for } \\
\text { each city in PRD }\end{array}$} \\
\hline & & & Artificial board & 0.5 & $\mathrm{~g} / \mathrm{kg}$ & \\
\hline & & \multirow{2}{*}{ Ink } & Printing & 320 & $\mathrm{~g} / \mathrm{kg}$ & $\begin{array}{c}\text { Guangdong Statistical } \\
\text { Yearbook }\end{array}$ \\
\hline & & & Printed circuit board & 0.093 & $\mathrm{~kg} / \mathrm{m}^{2}$ & $\begin{array}{c}\text { Statistical Yearbook for } \\
\text { each city in PRD }\end{array}$ \\
\hline & & Others & Personal domestic product & 0.5 for urban, 0.1 for rural & $\mathrm{kg} /$ capita & $\begin{array}{c}\text { Guangdong Statistical } \\
\text { Yearbook } 2011\end{array}$ \\
\hline Sun et al. (2018) & 2009-2013, China & Coating & Interior wall paint & 80 & $\mathrm{~g} / \mathrm{kg}$ paint & \\
\hline
\end{tabular}




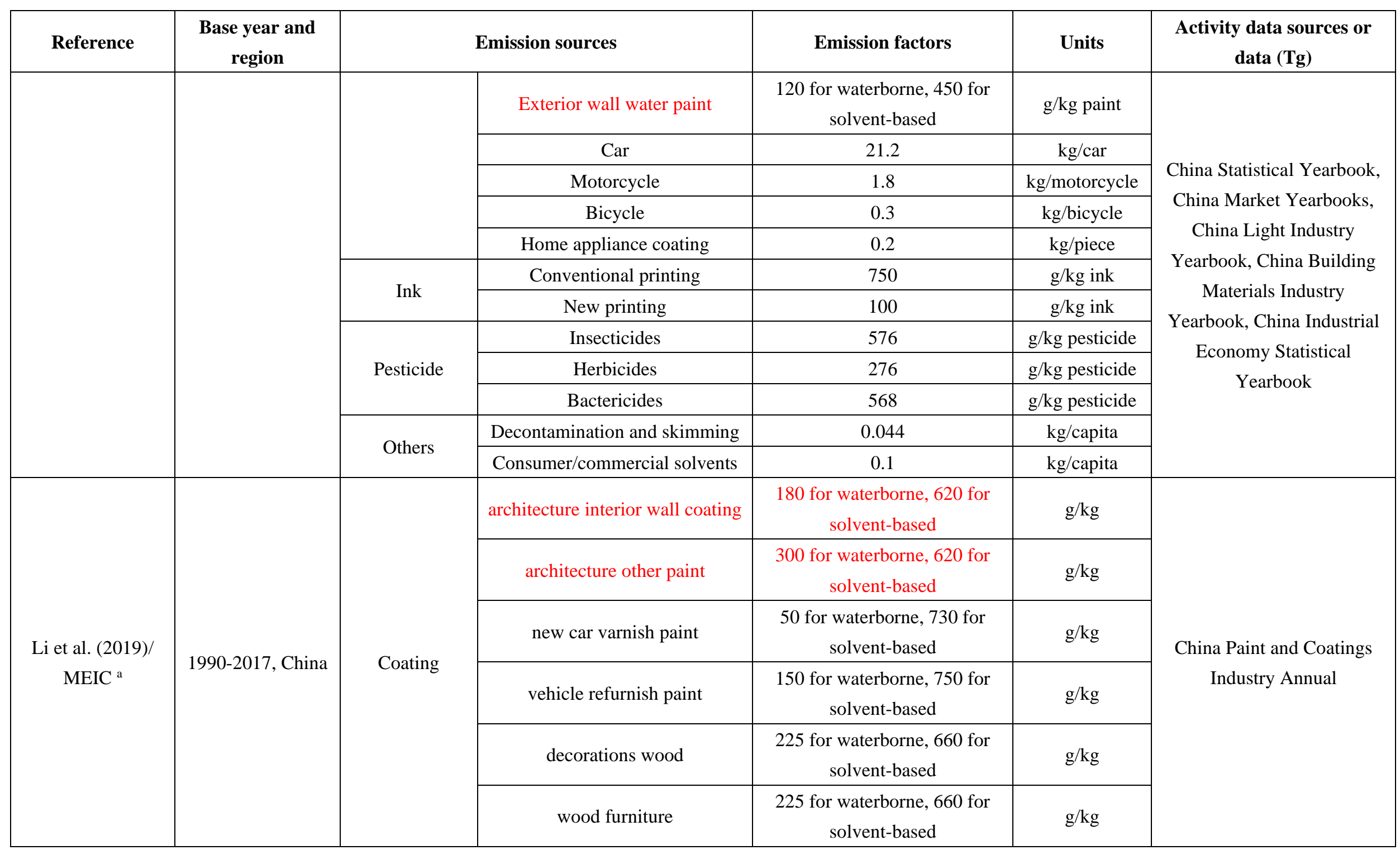




\begin{tabular}{|c|c|c|c|c|c|c|}
\hline Reference & $\begin{array}{c}\text { Base year and } \\
\text { region }\end{array}$ & \multicolumn{2}{|c|}{ Emission sources } & \multirow{2}{*}{$\begin{array}{c}\text { Emission factors } \\
440\end{array}$} & \multirow{2}{*}{$\begin{array}{l}\text { Units } \\
\mathrm{g} / \mathrm{kg}\end{array}$} & \multirow[t]{2}{*}{$\begin{array}{c}\text { Activity data sources or } \\
\text { data }(\mathrm{Tg})\end{array}$} \\
\hline & & & other industry paint & & & \\
\hline & & Adhesive & glue use & 66 & $\mathrm{~g} / \mathrm{kg}$ & $\begin{array}{c}\text { China Council for the } \\
\text { Promotion of International } \\
\text { Trade (CCPIT) }\end{array}$ \\
\hline & & Ink & printing ink & 540 & $\mathrm{~g} / \mathrm{kg}$ & China Statistical Yearbook \\
\hline
\end{tabular}

a. Emission factors in Li et al. (2019) are unabated emission factor without NMVOCs control. 

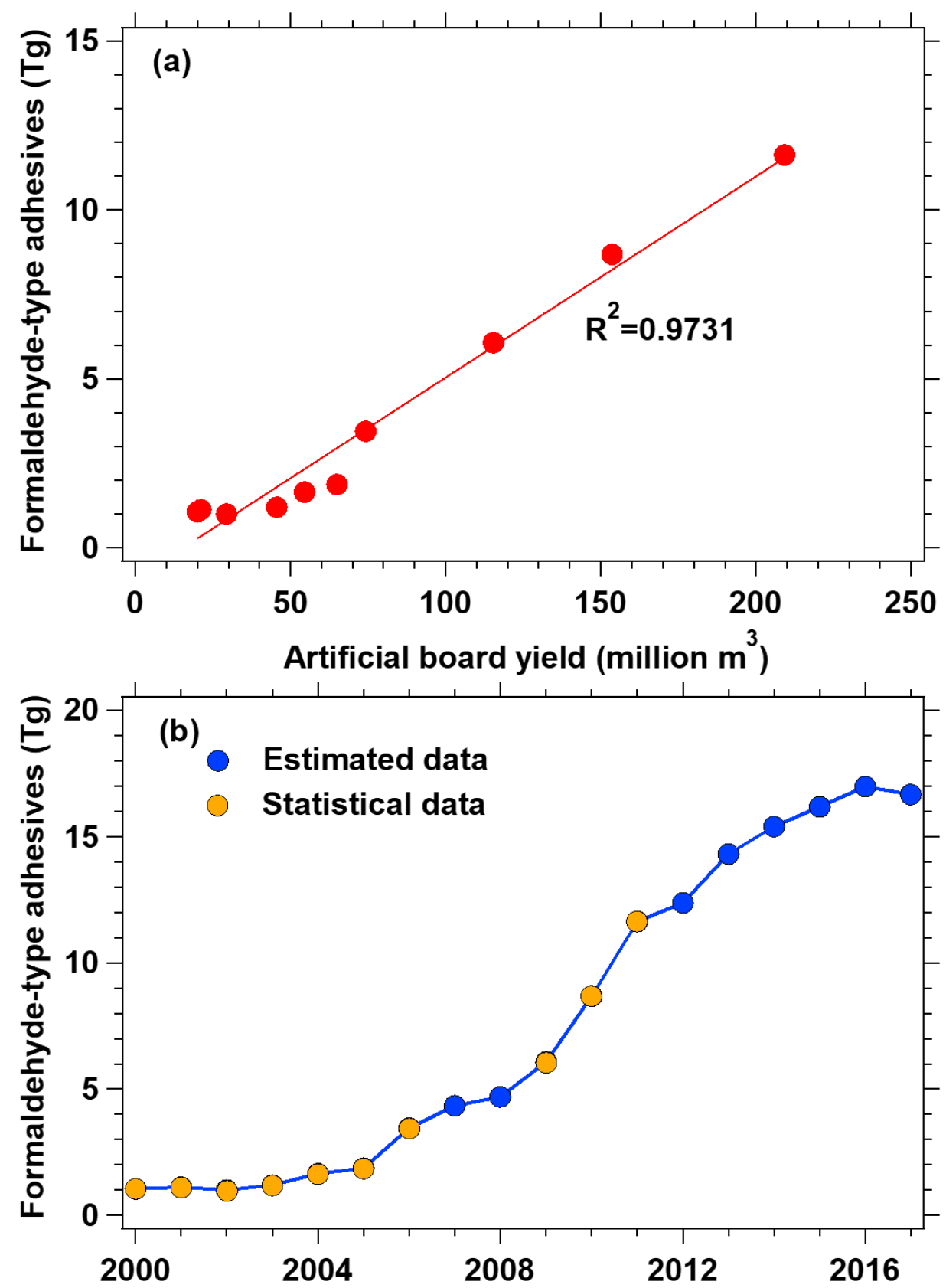

Figure S1. (a) The linear relationship between formaldehyde-type adhesive consumption and the artificial board yield for estimating consumption of formaldehyde-type adhesive, and (b) consumption of formaldehyde-type adhesive during 2000-2017. 


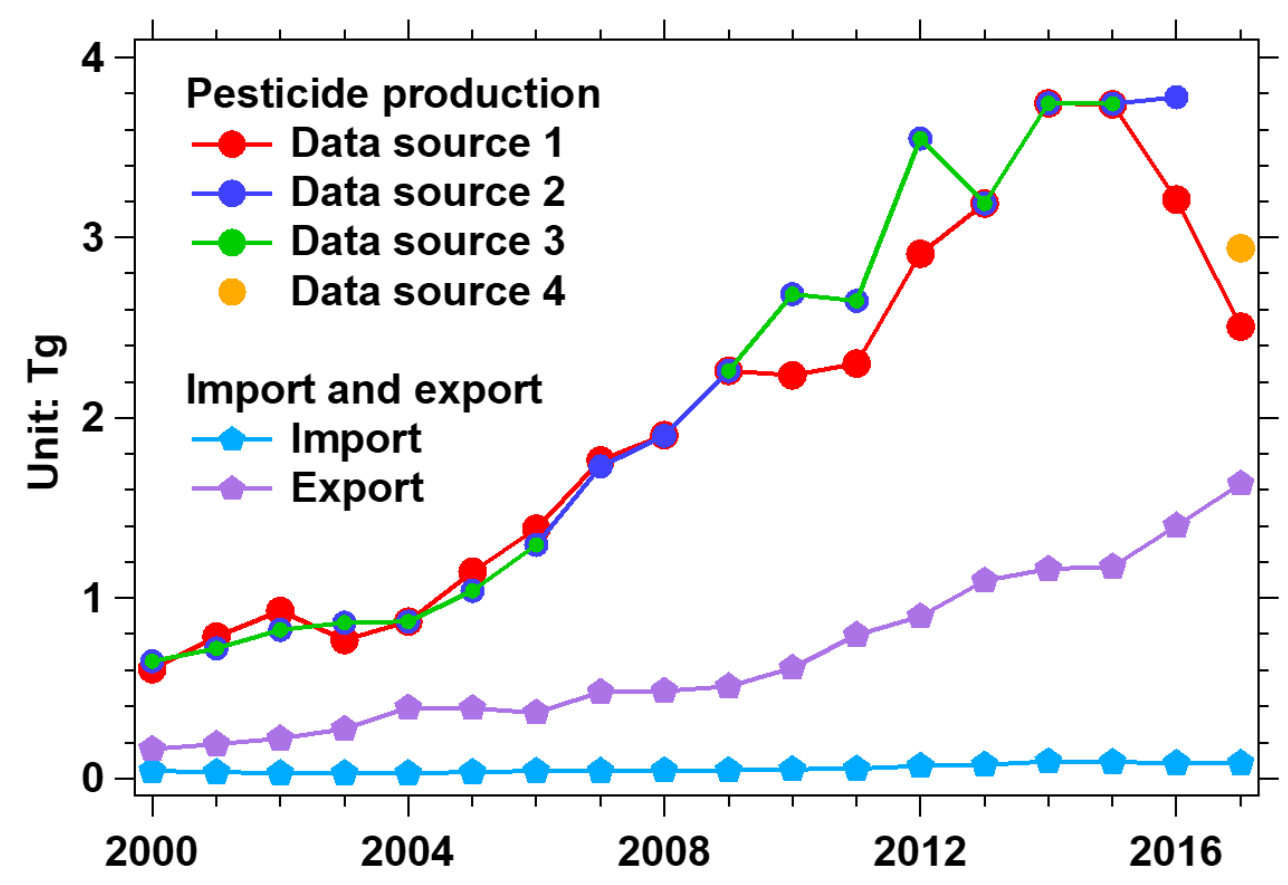

Figure S2. Pesticide production, import and export data during 2000-2017 in China. Data source 1: China Statistical Yearbook, missing import and export data; Data source 2: China Crop Protection Industry Yearbook; Data source 3: China Chemical Industry Yearbook; Data source 4: Duan (2018). 


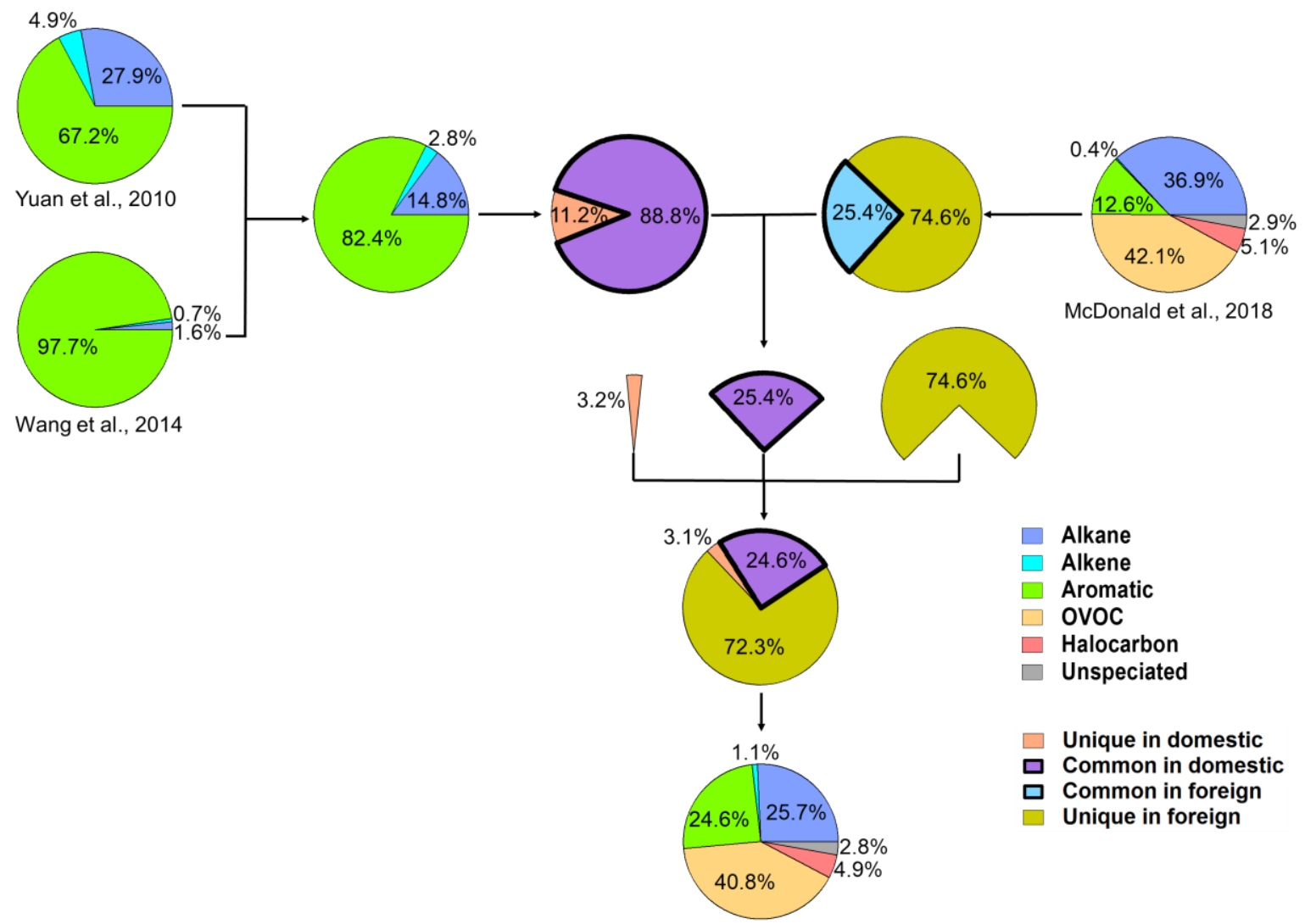

Figure S3. The procedure of obtaining merged source profiles for architectural coating. 


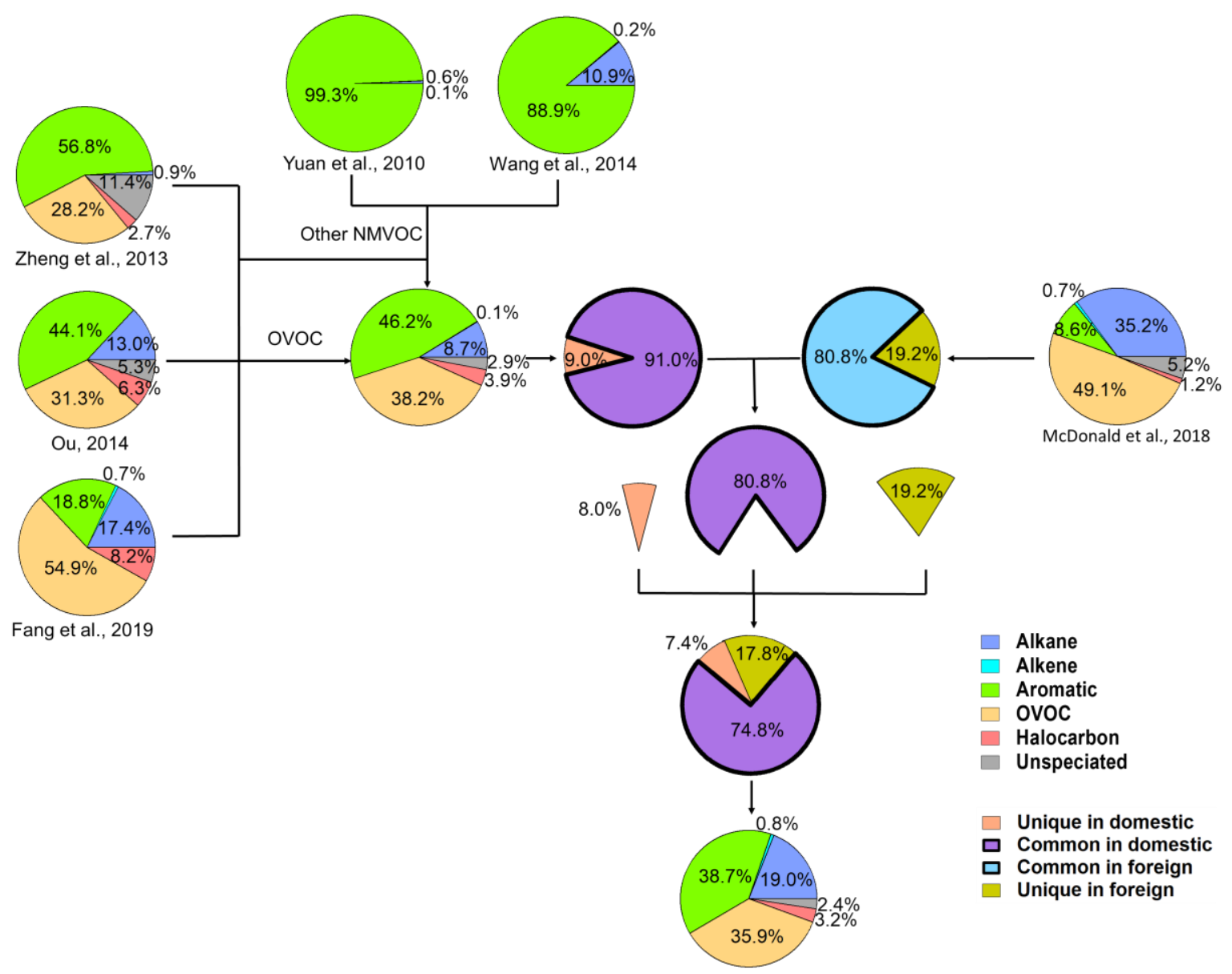

Figure S4. The procedure of obtaining merged source profiles for furniture coating. 


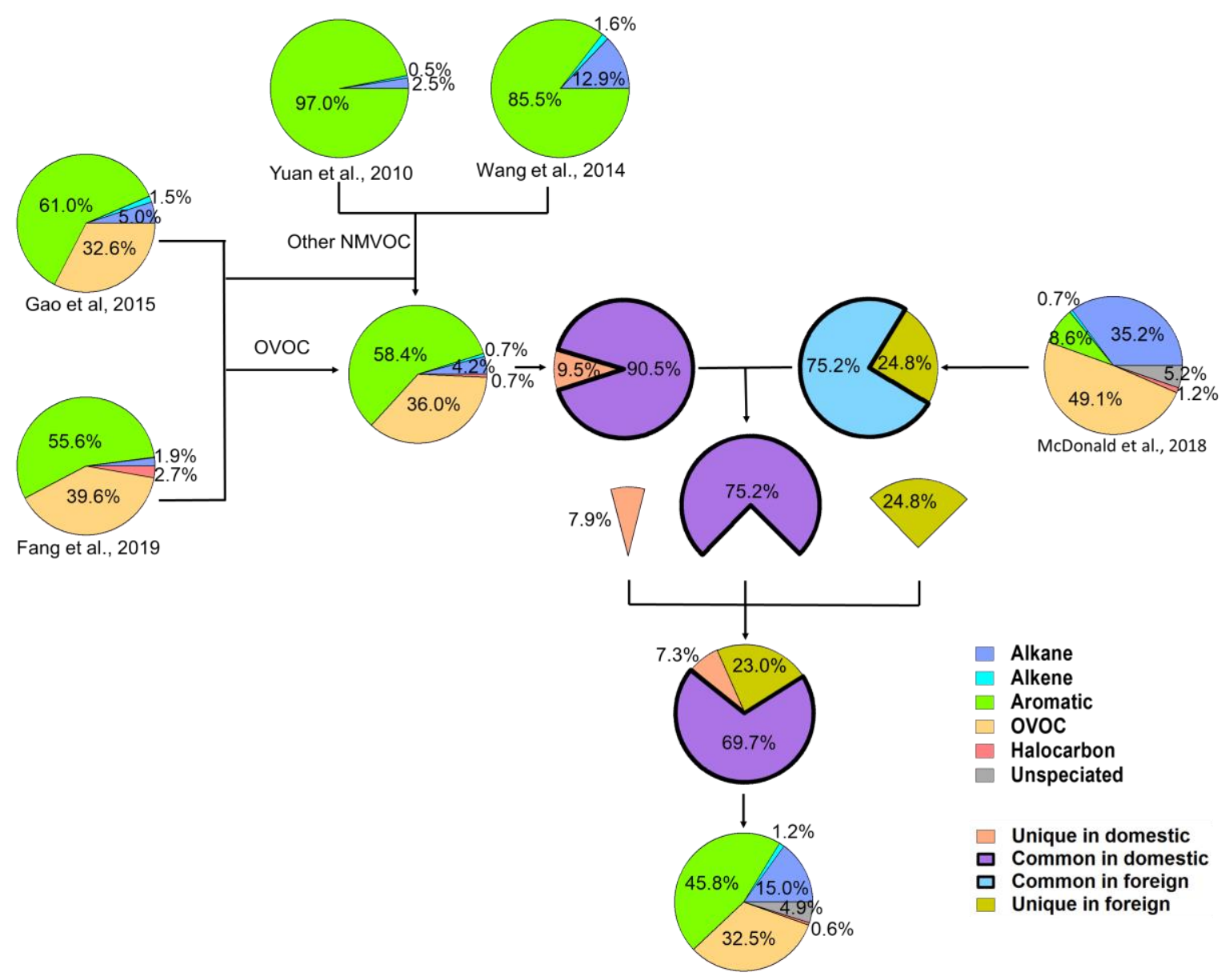

Figure S5. The procedure of obtaining merged source profiles for automobile coating. 


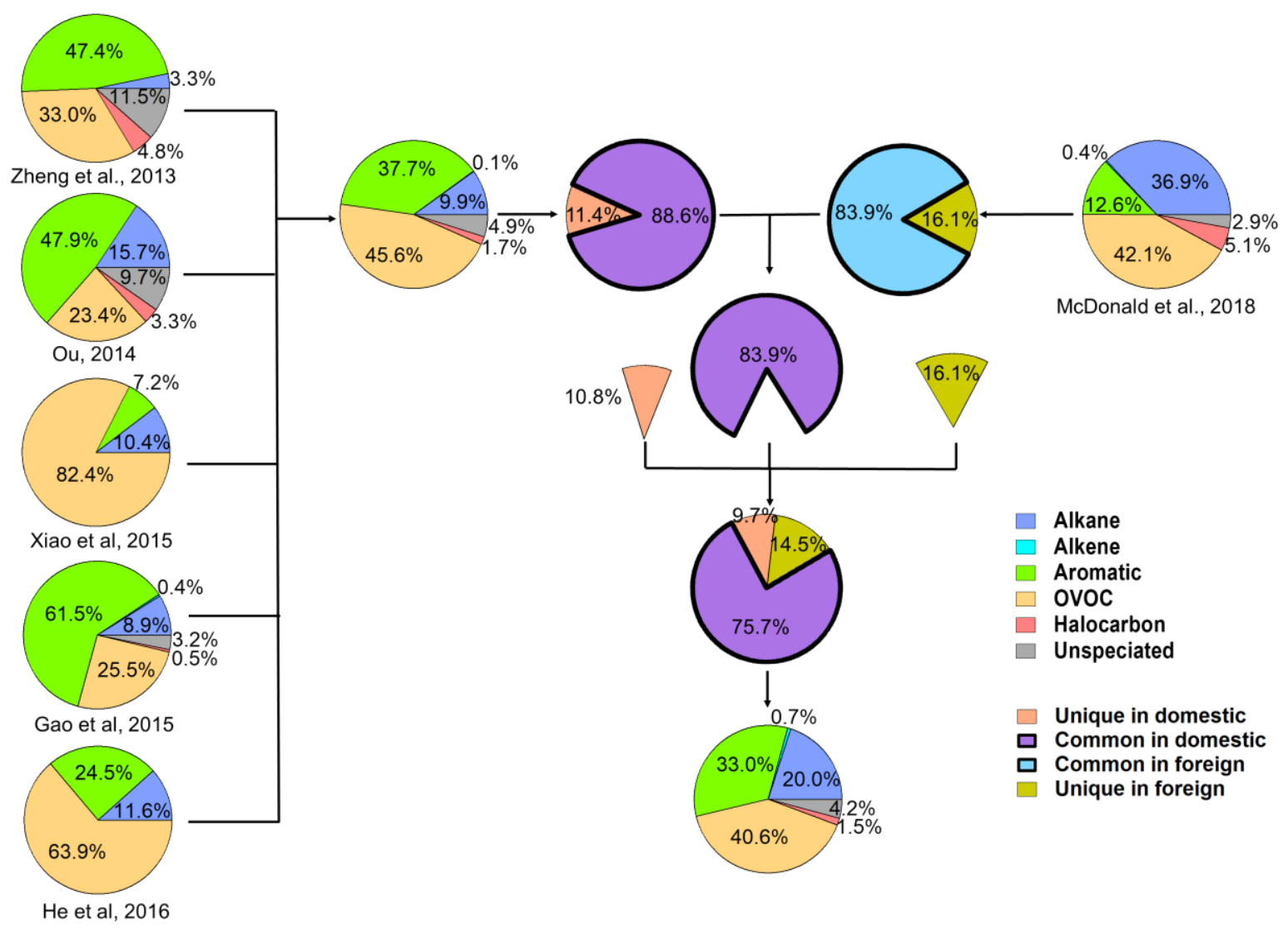

Figure S6. The procedure of obtaining merged source profiles for other industrial coating. 


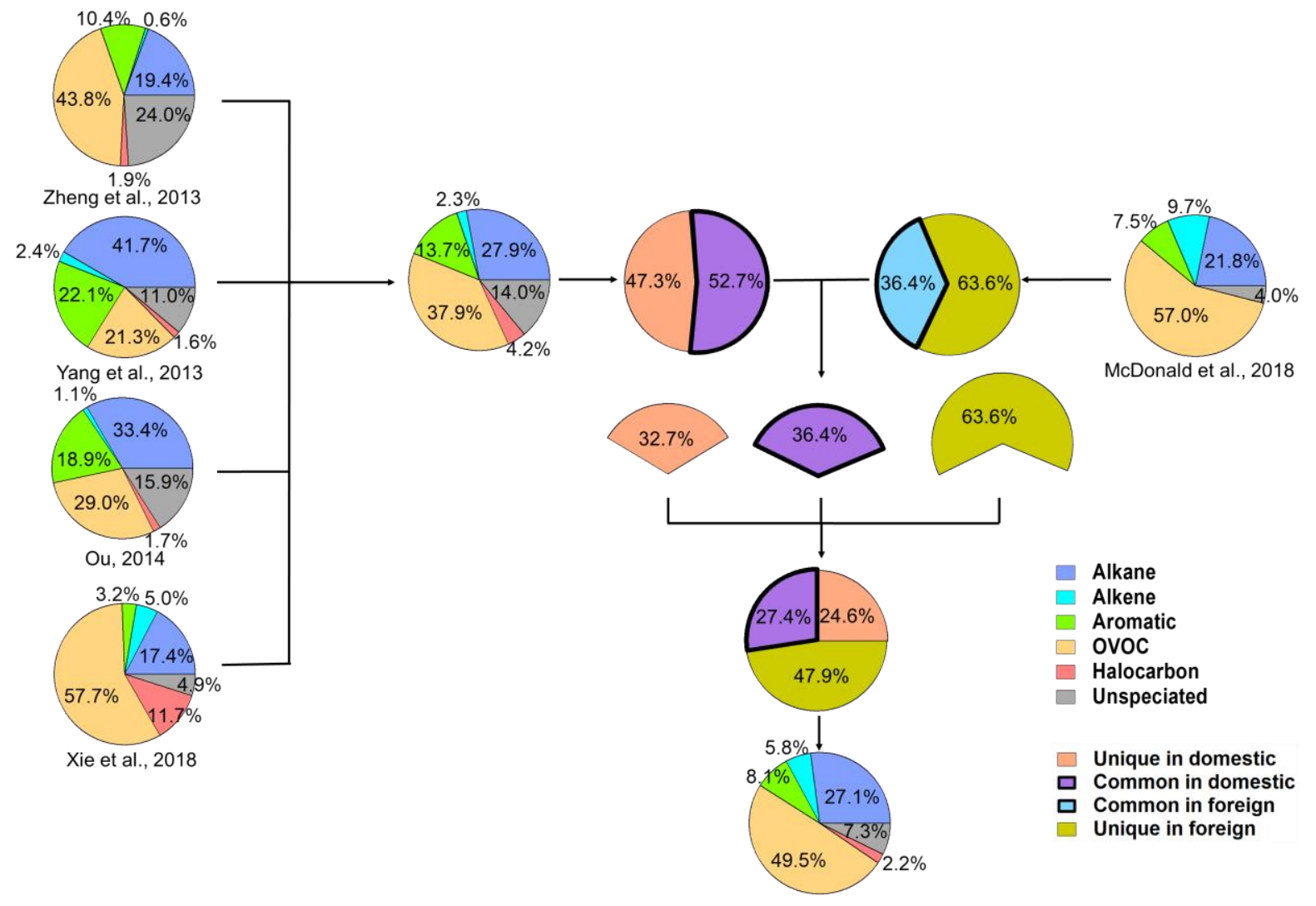

Figure S7. The procedure of obtaining merged source profiles for offset printing ink. 


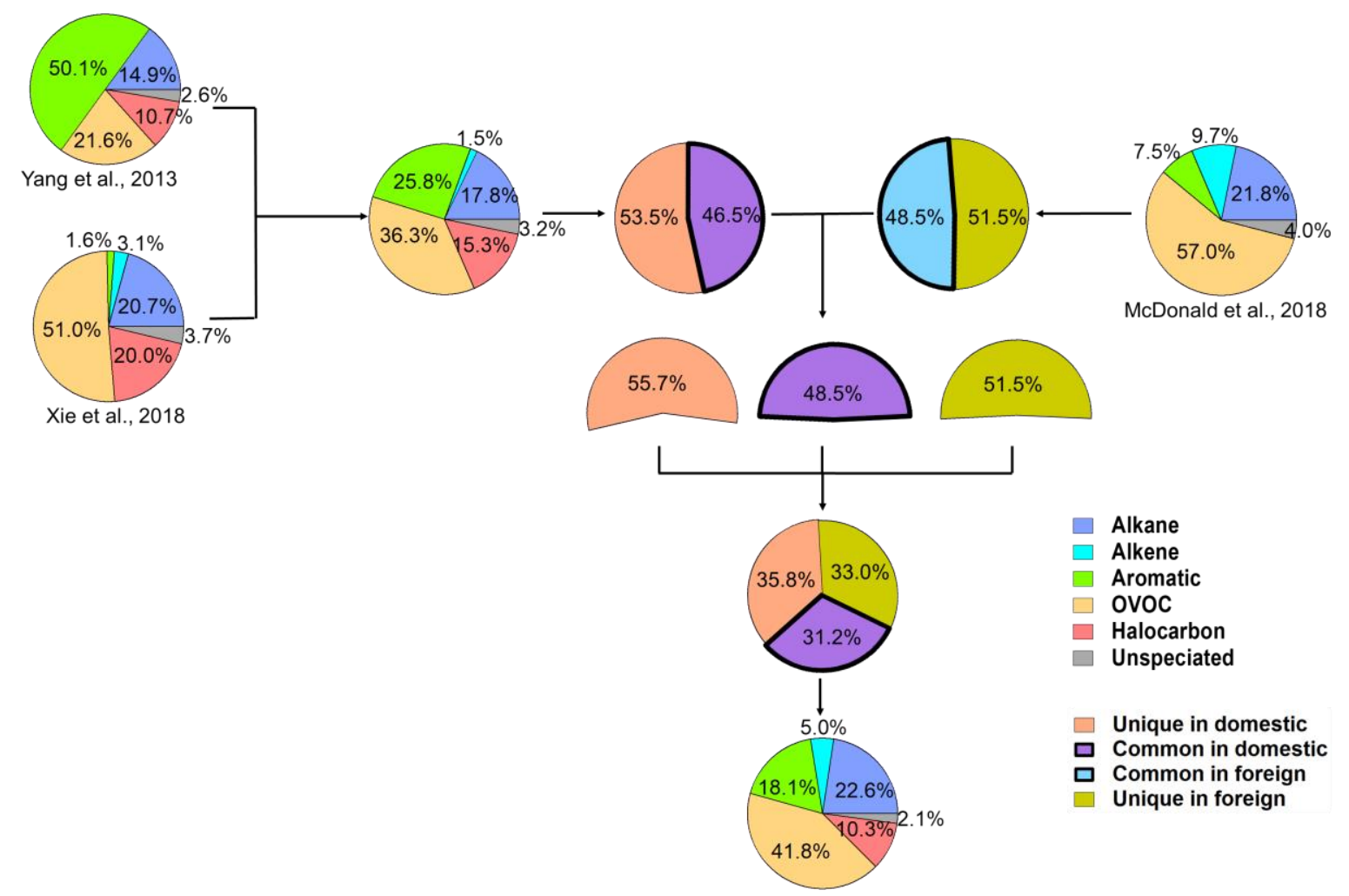

Figure S8. The procedure of obtaining merged source profiles for letterpress printing ink. 


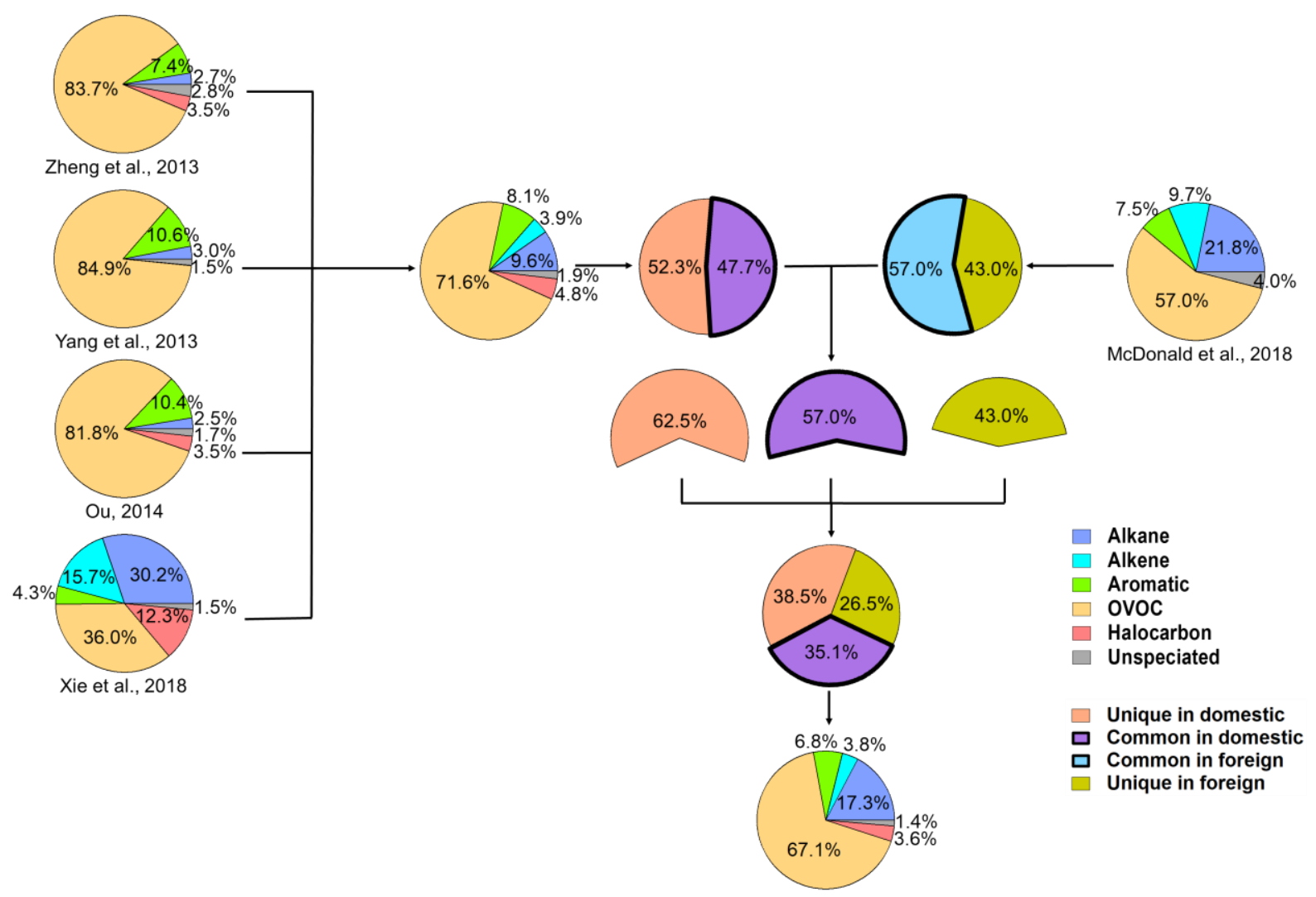

Figure S9. The procedure of obtaining merged source profiles for gravure printing ink. 


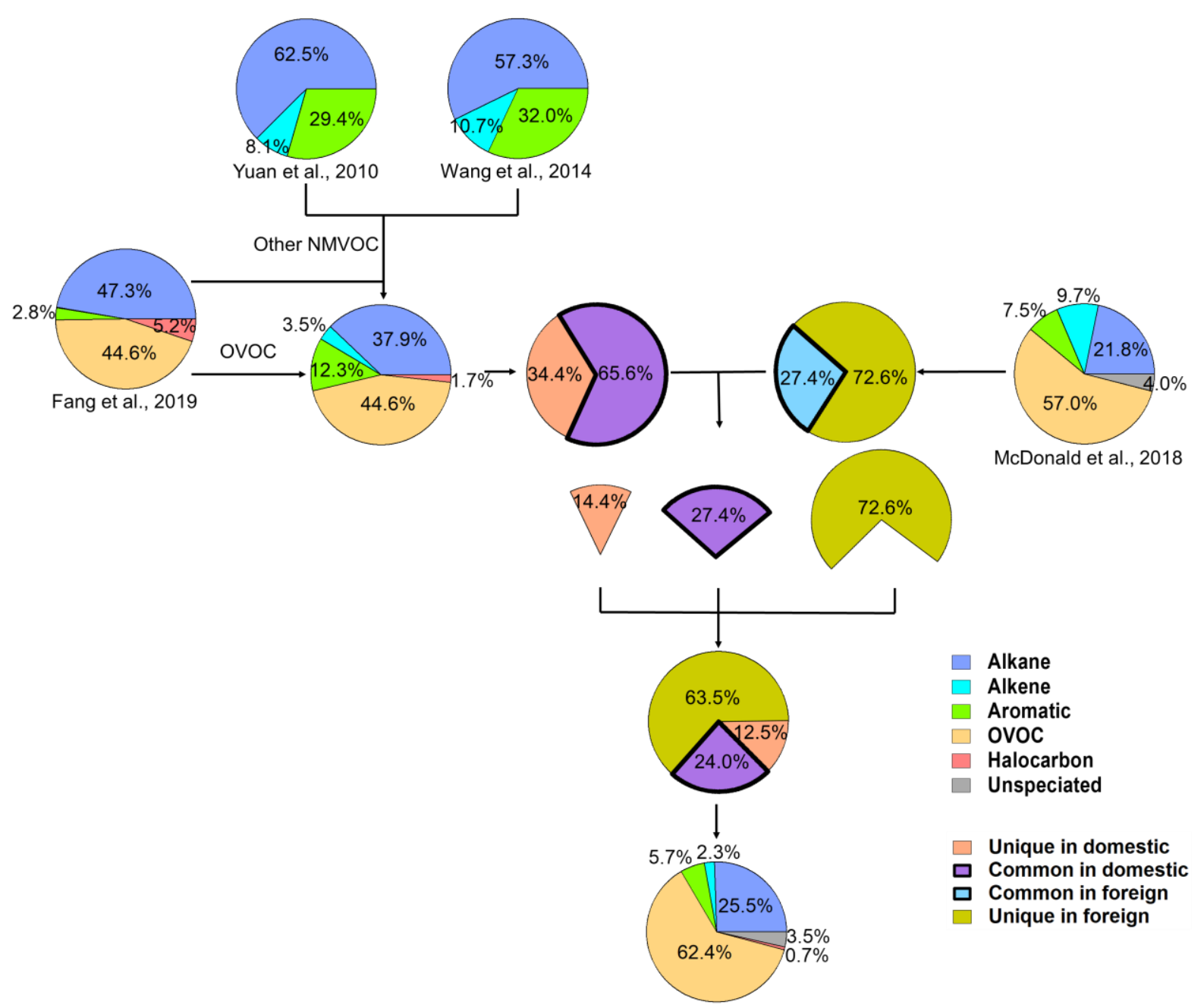

Figure S10. The procedure of obtaining merged source profiles for other printing ink. 


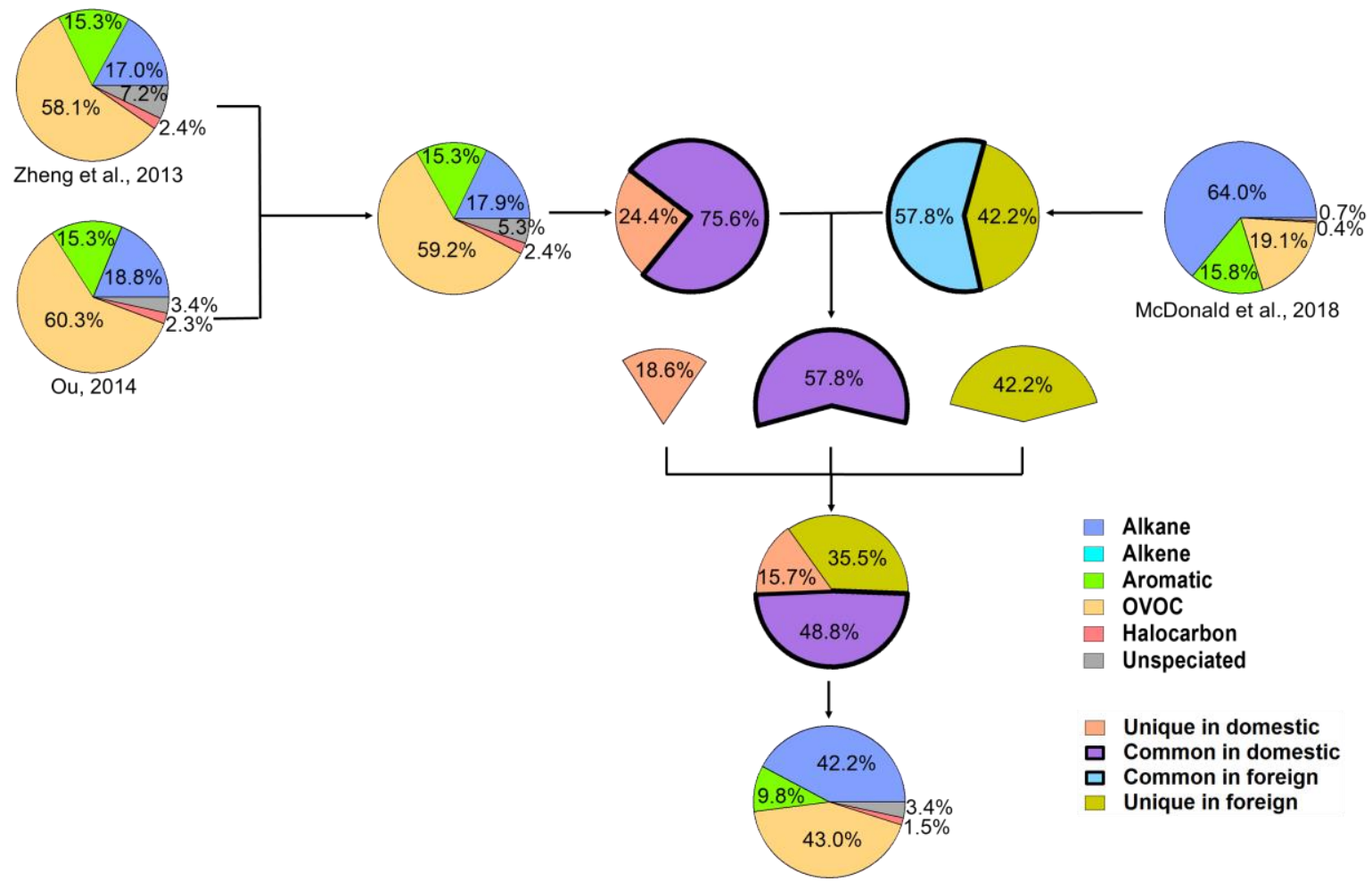

Figure S11. The procedure of obtaining merged source profiles for shoemaking adhesive. 


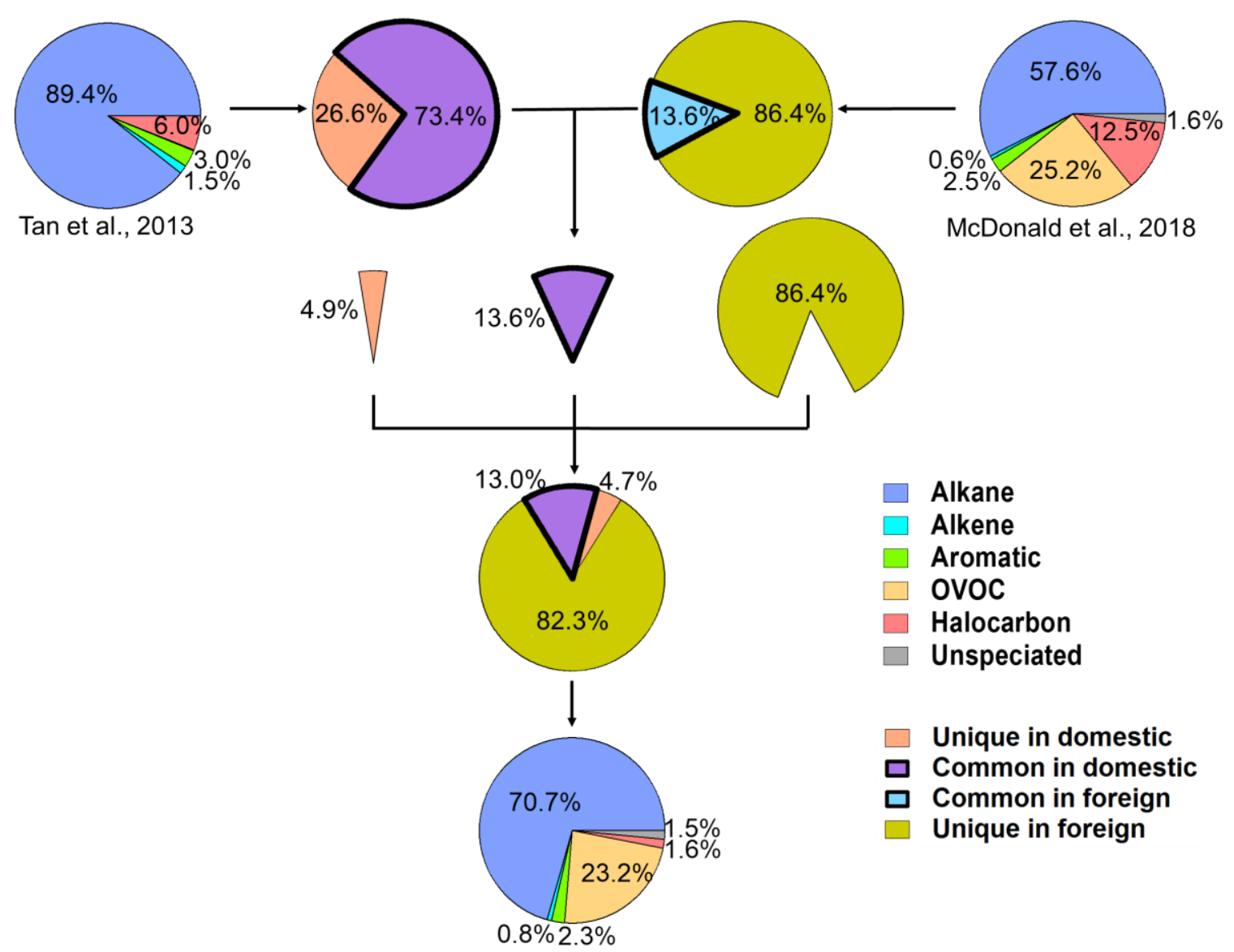

Figure S12. The procedure of obtaining merged source profiles for herbicide. 


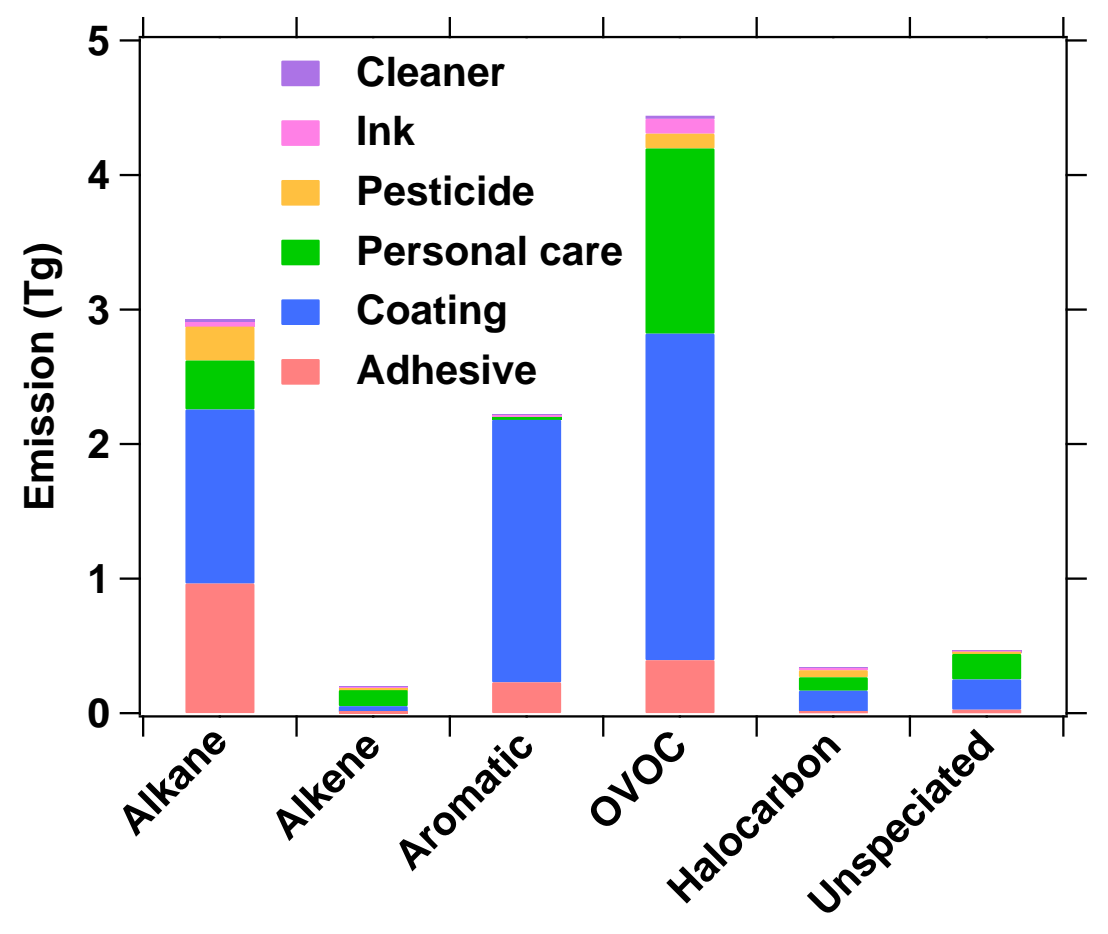

Figure S13. NMVOCs group emissions from various solvent categories in 2017.
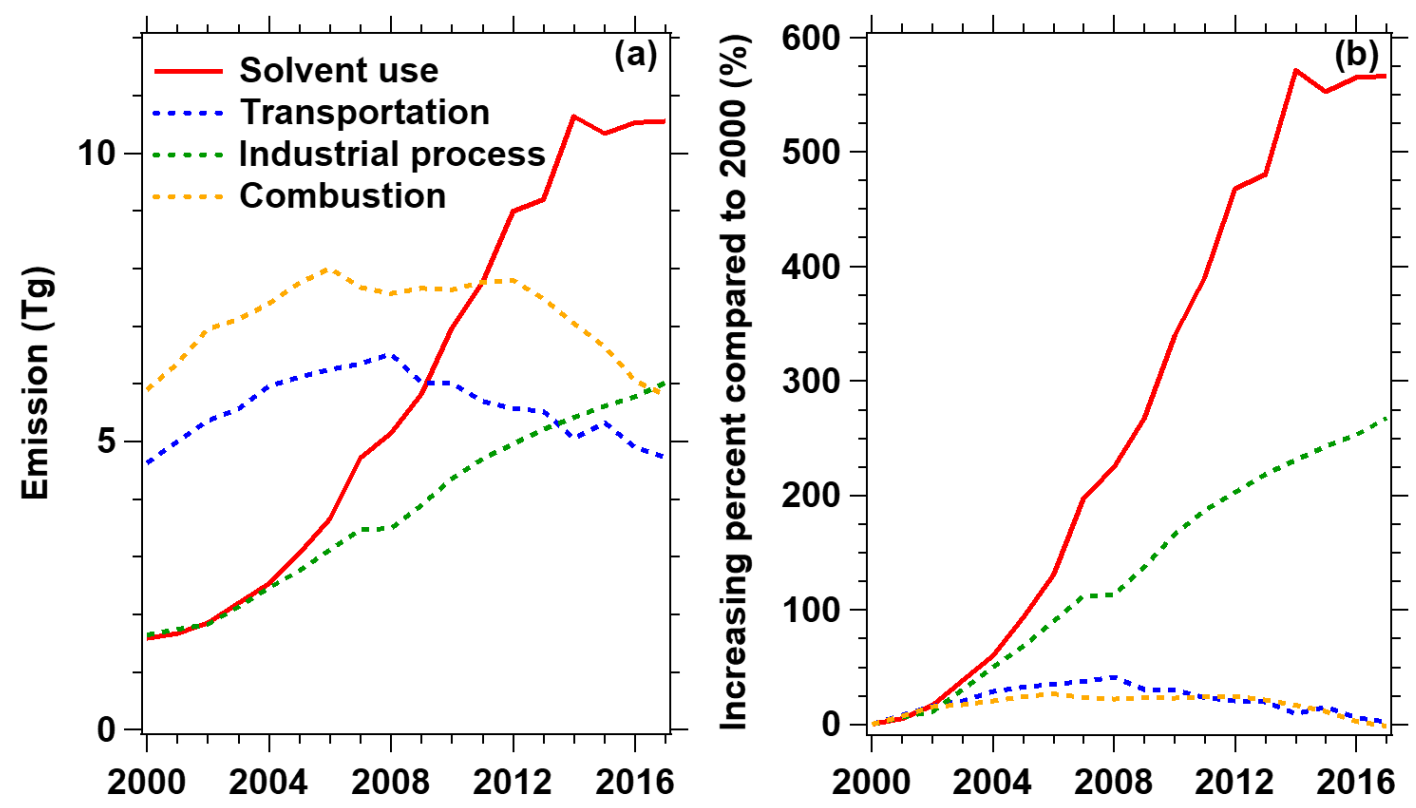

Figure S14. Comparisons of (a) NMVOCs emissions and (b) their increasing percentage compared to 2000 from solvent use (this study) and other sources (MEIC). 


\section{Reference}

Bai, Shan, and Zi, Yin: Water-based ink market (in Chinese), China Printing Materials Market, 27-28, 2003. Beijing Municipal Ecology and Environment Bureau: Detailed rules for the collection of pollutant discharge fees for volatile organic compounds, available at:

http://sthjj.beijing.gov.cn/bjhrb/index/xxgk69/zfxxgk43/fdzdgknr2/hbjfw/604447/index.html, 2015.

Bo, Y., Cai, H., and Xie, S. D.: Spatial and temporal variation of historical anthropogenic NMVOCs emission inventories in China, Atmospheric Chemistry and Physics, 8, 7297-7316, 10.5194/acp-8-7297-2008, 2008.

Cai, Huihua: The progress of pollution control on stationary sources in Guangdong Province, Guangdong Polytechnic of Environmental Protection Engineering, Foshan, China, 2016.

California air resources board: Regulation for Reducing Emissions from Consumer Products, available at: https://ww3.arb.ca.gov/consprod/regs/2019/article_2 final_5_2019.pdf, 2019a.

California air resources board: Regulation for Reducing VOC Emissions from Antiperspirants and Deodorants, available at: https://ww3.arb.ca.gov/consprod/regs/2019/article_1_final_5_2019.pdf, 2019b.

China National Coatings Industry Association: The 13th Five-year Plan in Coatings Industry in China, China Coatings, 31, 1-12, 2016.

Chyxx: Competition pattern and demand market Outlook of Waterborne coatings industry in China in 2014, available at: http://www.chyxx.com/industry/201408/275519.html, 2014.

Chyxx: Analysis of the market demand for shoe adhesive in China in 2014, available at: http://www.chyxx.com/industry/201508/335107.html, 2015.

Chyxx: Ink industry market development status and future development trend analysis in 2016, available at: http://www.chyxx.com/industry/201702/494485.html, 2017.

Department of Ecology and Environment of Guangdong Province: Calculation method of volatile organic compounds emission in key industries, available at: http://gdee.gd.gov.cn/shbtwj/content/post_2305668.html, 2019.

Department of Ecology and Environment of Jiangsu Province: Interim Measures of Jiangsu Province for the Calculation of emissions of Volatile organic compounds in key Industries, available at: http://hbt.jiangsu.gov.cn/art/2016/7/8/art_1581_6691890.html, 2016.

Department of Ecology and Environment of Shandong Province: Reference value of organic material type and VOCs content, available at: http://xxgk.sdein.gov.cn/zfwj/lhh/201705/t20170508 1173844.html, 2017.

Department of Ecology and Environment of Zhejiang Province: Interim Method for the Calculation of Volatile Organic compounds (VOCs) emissions from industrial coating Process and Printing Industry, available at: http://sthjt.zj.gov.cn/art/2017/6/26/art_1229263559_2258845.html, 2017.

Duan, Yousheng: China's pesticide industry in 2017 (in Chinese), Economic Analysis of China Petroleum and Chemical Industry, 47-49, 2018.

DYQ: Urea formaldehyde resin details, available at: https://www.dyq.cn/show/42001485.html, 2019.

Fang, Li, Liu, Wen-wen, Chen, Dan-ni, Li, Guo-hao, Wang, Di, Shao, Xia, and Nie, Lei: Source Profiles of Volatile Organic Compounds(VOCs) from Typical Solvent-based Industries in Beijing (in Chinese), Environmental Science, 40, 4395-4403, 2019.

Gao, Zongjiang: Source chareteristics of VOC emission from typical industrial painting sources (MS thesis). South China University of Technology, 2015.

Green Seal: GS-50 Personal Care and Cosmetic Products, available at: https://greenseal.org/green-sealstandards/gs-50, 2020.

He, Menglin, Wang, Ni, Chen, Yangda, Wu, Zeng, Chen, Jiandong, Fan, Liya, and Ye, Daiqi: Emission characteristics of volatile organic compounds( VOCs) from electronic manufacturing factory of Guangdong 
Province (in Chinese), Acta Scientiae Circumstantiae, 36, 1581-1588, 2016.

Henan River Grinding Material Co LTD: Phenolic resin details, available at:

http://www.hnbhsy.com/photo/667.html;http://www.hnbhsy.com/photo/649.html, 2020.

Huaon: The current situation of Waterborne coatings industry in China in 2018, available at:

https://www.huaon.com/story/446158, 2019.

Human Settlements and Environment Commission of Shenzhen Municipality: Limit of volatile organic compounds of industrial production and consumer goods available at:

http://meeb.sz.gov.cn/attachment/0/321/321040/2039498.pdf, 2016.

Li, M., Zhang, Q., Streets, D. G., He, K. B., Cheng, Y. F., Emmons, L. K., Huo, H., Kang, S. C., Lu, Z., Shao, M., Su, H., Yu, X., and Zhang, Y.: Mapping Asian anthropogenic emissions of non-methane volatile organic compounds to multiple chemical mechanisms, Atmospheric Chemistry and Physics, 14, 5617-5638, 10.5194/acp-14-5617-2014, 2014.

Li, Meng, Zhang, Qiang, Zheng, Bo, Tong, Dan, Lei, Yu, Liu, Fei, Hong, Chao Peng, Kang, Si Cong, Yan, Liu, Zhang, Yu Xuan, Bo, Yu, Su, Hang, Cheng, Ya Fang, and He, Ke Bin: Persistent growth of anthropogenic nonmethane volatile organic compound (NMVOC) emissions in China during 1990-2017: drivers, speciation and ozone formation potential, Atmospheric Chemistry and Physics, 19, 8897-8913, 10.5194/acp-19-8897-2019, 2019.

Liu, Guojie: Current situation and development trend of architectural coatings (in Chinese), Architecture Decoration, 6-7, 2000.

Lu, Jianhai, Dong, Shibi, Li, Wenjuan, Miu, Xiaoping, and Gu, Zhenyu: Present Situation of VOCs Control Technologies for the Industrial Coating Process in Zhejiang Province (in Chinese). Environmental Protection Science, 44, 113-117+121, 2018.

McDonald, Brian C., de Gouw, Joost A., Gilman, Jessica B., Jathar, Shantanu H., Akherati, Ali, Cappa, Christopher D., Jimenez, Jose L., Lee-Taylor, Julia, Hayes, Patrick L., McKeen, Stuart A., Cui, Yu Yan, Kim, SiWan, Gentner, Drew R., Isaacman-VanWertz, Gabriel, Goldstein, Allen H., Harley, Robert A., Frost, Gregory J., Roberts, James M., Ryerson, Thomas B., and Trainer, Michael: Volatile chemical products emerging as largest petrochemical source of urban organic emissions, Science, 359, 760, 10.1126/science.aaq0524, 2018.

National Bureau of Statistics: 2017 China Industrial Statistical Yearbook, China Statistics Press, Beijing, China, 2017.

National Bureau of Statistics: 2017 China Statistical Yearbook, China Statistics Press, Beijing, China, 2018. Ou, Jiaming: Characterization and validation of Volatile Organic Compounds (VOCs) emission sources and reactivity-based control strategy in the Pearl Delta region (MS thesis). South China University of Technology, 2014.

Qianzhan: A panoramic map of China's ink industry in 2019, available at:

https://www.qianzhan.com/analyst/detail/220/190618-27096aef.html, 2019.

Shanghai Municipal Bureau of Ecology and Environment: General method for calculating the emission of volatile organic compounds from industrial enterprises in Shanghai, available at:

https://sthj.sh.gov.cn/hbzhywpt1133/hbzhywpt1135/20170308/0024-103293.html, 2017.

Sun, W., Shao, M., Granier, C., Liu, Y., Ye, C. S., and Zheng, J. Y.: Long-Term Trends of Anthropogenic SO2, NOx, CO, and NMVOCs Emissions in China, Earth's Future, 6, 1112-1133, 10.1029/2018ef000822, 2018. VOCs.BJX: VOCs escape point, emission, concentration and characteristic pollutants in printing industry, available at: http://huanbao.bjx.com.cn/news/20190822/1001864.shtml, 2019. 
Vzkoo: Hydrocarbon environmental protection solvent huge market potential, available at: https://www.vzkoo.com/news/815.html, 2019.

Wang, Hongli, Qiao, Yuezhen, Chen, Changhong, Lu, Jun, Dai, Haixia, Qiao, Liping, Lou, Shengrong, Huang, Cheng, Li, Li, Jing, Shengao, and Wu, Jianping: Source Profiles and Chemical Reactivity of Volatile Organic Compounds from Solvent Use in Shanghai, China, Aerosol and Air Quality Research, 14, 301-310, 10.4209/aaqr.2013.03.0064, 2014.

Wei, W., Wang, S., and Hao, J.: Uncertainty analysis of emission inventory for volatile organic compounds from anthropogenic sources in China (in Chinese). Environmental Science, 32, 305-312, 2011a.

Wei, Wei, Wang, Shuxiao, Hao, Jiming, and Cheng, Shuiyuan: Projection of anthropogenic volatile organic compounds (VOCs) emissions in China for the period 2010-2020, Atmospheric Environment, 45, 6863-6871, https://doi.org/10.1016/j.atmosenv.2011.01.013, 2011b.

Wu, R., and Xie, S.: Spatial Distribution of Ozone Formation in China Derived from Emissions of Speciated Volatile Organic Compounds, Environ Sci Technol, 51, 2574-2583, 10.1021/acs.est.6b03634, 2017.

Xiao, Jingfang, Ye, Daiqi, Liu, Qiao, Peng, Ruosi, and Wang, Guannan: Emission characteristics of volatile organic compounds ( VOCs ) from the manufacturing process of consumer electronic products (in Chinese), Acta Scientiae Circumstantiae, 35, 1612-1619, 2015.

Xie, Yisong, Zheng, Xinmei, and Liu, Chunlei: Source Profiles and Ozone Formation Potental of Volatile Organic Compounds from Printing Industry in Nanjing (in Chinese), Environmental Science and Technology, 31, 64-67, 2018.

Yang, Yang, Yang, Jing, Yin, Shasha, Yu, Yufan, Zheng, Jun-yu, and Mo, Ziwei: Speciated VOCs Emission Inventory and Key Species from Printing Industry in the Pearl River Delta Region (in Chinese), Research of Environmental Sciences, 26, 326-333, 2013.

Yin, S., Zheng, J., Lu, Q., Yuan, Z., Huang, Z., Zhong, L., and Lin, H.: A refined 2010-based VOC emission inventory and its improvement on modeling regional ozone in the Pearl River Delta Region, China, Sci Total Environ, 514, 426-438, 10.1016/j.scitotenv.2015.01.088, 2015.

Yuan, Bin, Shao, Min, Lu, Sihua, and Wang, Bin: Source profiles of volatile organic compounds associated with solvent use in Beijing, China, Atmospheric Environment, 44, 1919-1926, 10.1016/j.atmosenv.2010.02.014, 2010. Yuan, Changbo, and Chen, Jincai: A new adhesive for shoes (in Chinese), China Adhesives, 32-33, 2005.

Zheng, J., Yu, Y., Mo, Z., Zhang, Z., Wang, X., Yin, S., Peng, K., Yang, Y., Feng, X., and Cai, H.: Industrial sector-based volatile organic compound (VOC) source profiles measured in manufacturing facilities in the Pearl River Delta, China, Sci Total Environ, 456-457, 127-136, 10.1016/j.scitotenv.2013.03.055, 2013. 